\title{
Trapped instability and vortex formation by an unstable coastal current
}

\author{
Rui Duarte ${ }^{1}$, Xavier Carton ${ }^{1}$, Xavier Capet $^{1}$ and Laurent Chérubin ${ }^{2}$
}

\author{
${ }^{1}$ LPO, UBO/CNRS, Brest, France \\ ${ }^{2}$ RSMAS, Univ. Miami, USA \\ *: Corresponding authors : Rui Duarte : Rui.Duarte@univ-brest.fr ; Xavier Carton : Xavier.Carton@univ-brest.fr ; \\ Xavier Capet : Xavier.Capet@ifremer.fr ; Laurent Chérubin : Icherubin@rsmas.miami.edu
}

\begin{abstract}
:
This paper addresses the instability of a two-layer coastal current in a quasigeostrophic model; the potential vorticity (PV) structure of this current consists in two uniform cores, located at different depths, with finite width and horizontally shifted. This shift allows both barotropic and baroclinic instability for this current. The PV cores can be like-signed or opposite-signed, leading to their vertical alignment or to their hetonic coupling. These two aspects are novel compared to previous studies. For narrow vorticity cores, short waves dominate, associated with barotropic instability; for wider cores, longer waves are more unstable and are associated with baroclinic processes. Numerical experiments were performed on the $f$-plane with a finite-difference model. When both cores have like-signed PV, trapped instability develops during the nonlinear evolution: vertical alignment of the structures is observed. For narrow cores, short wave breaking occurs close to the coast; for wider cores, substantial turbulence results from the entrainment of ambient fluid into the coastal jet. When the two cores have opposite-signed PV, the nonlinear regimes range from short wave breaking to the ejection of dipoles or tripoles, via a regime of dipole oscillation near the wall. The Fourier analysis of the perturbed flow is appropriate to distinguish the regimes of short wave breaking, of dipole formation, and of turbulence, but not the differences between regimes involving only vortex pairs. To explain more precisely the regimes where two vortices (and their wall images) interact, a point vortex model is appropriate.
\end{abstract}

Keywords : stability and instability of geophysical and astrophysical flows ; vortex flows ; rotating fluids ; stability problems ; applications to physics 


\section{INTRODUCTION}

Planetary fluids, strongly constrained by rotation and stratification, are a favorable medium for the birth, growth and interaction of intense vortices. In the ocean in particular, such vortices can be generated by the instability of intense, sheared currents. If intense vortices are often found in the upper layers of the ocean, others can grow and live for hundreds of turnover periods in the ocean depths.

A well-known example of surface current is the Kuroshio, which is the western boundary current of the North Pacific Ocean. South of Japan, the Kuroshio is a fast and narrow current flowing north-eastward along the continental slope, and making a deflection at about $138^{\circ} \mathrm{E}$, giving rise to the Kuroshio extension (KE) east of Japan. The instability of this current produces many vortices, and indeed, this region has the highest turbulent kinetic energy in the North Pacific ([16]).

From the WOCE database, we selected section P10 with CTD/LADCP stations across the path of the Kuroshio. We used temperature and salinity data to calculate the density. From the velocity, we computed the relative vorticity and finally we obtained Ertel potential vorticity $(\mathrm{PV})^{1}$. Here, we present the Ertel PV anomalies (PV of the current minus PV of the environment), which characterise the structure of the Kuroshio. We obtain a two-layer distribution with nearly uniform cores, laterally shifted, with opposite signs, and thickness of about $200 \mathrm{~m}$ (see figure 1).

A famous example of a deep coastal current is the Mediterranean Water undercurrent, which exits from the Mediterranean Sea through the Straits of Gibraltar, and then follows the Portuguese continental slope. South of Portugal, this current is composed of two cores, near 800 and $1200 \mathrm{~m}$ depths. It is known to produce many vortices, called meddies. These two cores have negative anomalies of Ertel PV compared to their environment ([5]). Measurements at sea show several configurations for the position of these PV cores: they can be vertically aligned or laterally shifted (see figure 5 of [6]).

The baroclinic instability of a coastal current, constituted of two PV cores vertically aligned, has been addressed previously ([2]; [1]; [7]). For vertically shifted cores, both barotropic and baroclinic instabilities are possible, whatever the sign of each PV anomaly.

\footnotetext{
${ }^{1}$ Ertel potential vorticity was computed here as the product of absolute vorticity by the vertical density gradient. Absolute vorticity is the sum of the Coriolis parameter and of relative vorticity.
} 
The objective of the present paper is to determine the conditions of prevalence of either type of instability (barotropic or baroclinic), the ensuing nonlinear regimes in parameter space, and to quantify them with simple models. In section 2, we present the model and physical configuration for this problem. In section 3, we provide the mathematical analysis of linear stability of the current. In section 4 , we classify and describe the nonlinear regimes of the unstable current, for both like-signed and opposite-signed PV cores. Finally, in section 5, we discuss more completely the regimes of vortex dipole oscillation or ejection.

\section{MODEL AND CONFIGURATION}

We consider a two-layer quasi-geostrophic model for ocean currents. This model assumes that the Rossby number of the flow $R o=U / f L$ is small, and that its Burger number $B u=(N H / f L)^{2}$ is of order unity. $U$ is a characteristic velocity magnitude, $L$ a characteristic width, $H$ a characteristic thickness, $f$ is the Coriolis parameter (twice the rotation rate of the Earth, times the sine of the latitude), and $N$ is the buoyancy frequency. Here, we neglect the variations of the Coriolis parameter with latitude, which are small compared with the PV of the flow.

The evolution of the fluid flow in this model is governed by the following equation

$$
\partial_{t} q_{k}+J\left(\psi_{k}, q_{k}\right)=D_{k} \quad(k=1,2)
$$

which describes the advection and dissipation of quasi-geostrophic potential vorticity. Quasigeostrophic potential vorticity is the equivalent of Ertel potential vorticity anomalies under the conditions $R o \ll 1, B u \sim 1$. In the upper and lower layers, quasi-geostrophic potential vorticity is defined by

$$
\begin{aligned}
& q_{1}=\nabla^{2} \psi_{1}-F_{1}\left(\psi_{1}-\psi_{2}\right) \\
& q_{2}=\nabla^{2} \psi_{2}-F_{2}\left(\psi_{1}-\psi_{2}\right)
\end{aligned}
$$

We also define $J(a, b)=\partial_{x} a \partial_{y} b-\partial_{x} b \partial_{y} a$, the Jacobian of $a$ and $b$, and $\psi_{k}$ is the stream function in layer $k$. The Froude numbers are $F_{n}=f_{0}^{2} L^{2} /\left(g^{\prime} H_{n}\right)$ for each layer $(j=1,2)$, $H_{n}$ is the nth layer depth at rest, $f_{0}$ is the Coriolis parameter and $g^{\prime}=g\left(\rho_{2}-\rho_{1}\right) / \rho_{1}$ is the reduced gravity between the layers. The dissipation terms $D_{n}$ exist only in the nonlinear numerical model (see below). 
To follow the example of the Kuroshio, we set $H_{1}=H_{2}=200 \mathrm{~m}, f_{0}=10^{-4} \mathrm{~s}^{-1}$, and $g^{\prime}=9 \times 10^{-2} \mathrm{~ms}^{-2}$, which corresponds to a typical ocean stratification in the Kuroshio region. The Rossby deformation radius is defined by

$$
R_{d}=\sqrt{\frac{g^{\prime} H_{1} H_{2}}{f_{0}^{2}\left(H_{1}+H_{2}\right)}}
$$

In our configuration the value of the Rossby radius is $R_{d}=30 \mathrm{~km}$.

For nonlinear evolutions, equations 1 and 2 are solved using a finite-difference code which uses an Arakawa energy and enstrophy conserving scheme. The model domain is a flat bottom periodic channel, with along-shore $x$ and cross-shore $y$ coordinates. The horizontal resolution is $\Delta x=\Delta y=5 \times 10^{3} m$ on a $200 \times 100$ grid. In the numerical model, the dissipation terms $D_{n}$ represent bi-harmonic diffusion which suppressed the accumulation of grid scale vorticity features; specifically,

$$
D_{n}=\nu \nabla^{4}\left(\nabla^{2} \psi_{n}\right)
$$

where $\nu=10^{8} \mathrm{~m}^{4} \mathrm{~s}^{-1}$ is the bi-harmonic diffusion coefficient. This model is initialised with the mean flow (the equivalent of the Kuroshio current) slightly perturbed with a sinusoidal wave.

The mean flow has a piecewise-constant PV distribution in both layers, with values $Q_{1}$ and $Q_{2}$, respectively. The lower $\mathrm{PV}$ core lies against the coast and the upper PV core is offshore. The current width is $L_{1}$ in the lower layer and $L=L_{2}-L_{1}$ in the upper layer (see figure 2).

$$
q_{1}(y)=\left\{\begin{array}{ll}
Q_{1} & \text { if } y \in\left[L_{1}, L_{2}\right] \\
0 & \text { otherwise }
\end{array} \quad q_{2}(y)= \begin{cases}Q_{2} & \text { if } y \in\left[0, L_{1}\right] \\
0 & \text { otherwise }\end{cases}\right.
$$

In summary, there are two PV fronts in the upper layer (at $y=L_{1}$ and $y=L_{2}$ ) with jumps $\pm Q_{1}$ and one in the lower layer (at $y=L_{1}$ ) with jump $-Q_{2}$. The corresponding streamfunction field and velocity profiles are shown in figure 3 for particular values of $Q_{1}$, $Q_{2}, L_{1}$ and $L_{2}$.

\section{LINEAR STABILITY ANALYSIS}

To study the stability of the mean flow, we consider its perturbation by a weak normal mode associated with the streamfunction

$$
\psi_{j}^{\prime}=\phi_{j}(j) e^{i k(x-c t)}
$$


It is associated with meridional displacements of the PV fronts of the mean flow; these displacements are called $\left(\eta_{1}, \eta_{2}, \eta_{3}\right)=\left(\eta_{1}^{0}, \eta_{2}^{0}, \eta_{3}^{0}\right) e^{i k(x-c t)}$ in the upper and lower layers respectively. To solve the linear stability problem, three conditions are used:

1. the perturbation has vanishing potential vorticity;

2. the perturbation streamfunction is continuous at each PV front of the mean flow;

3. the perturbation streamfunction is related to the PV front displacements via the continuity of the total zonal velocity (mean flow + perturbation).

4. the Lagrangian derivative of the PV front displacement is equal to the meridional perturbation velocity at the PV front

Finally, these equations are linearised around the zonal mean flow with the normal mode perturbation (or, in an equivalent manner,the dynamical equations 1 and 2 are linearised). The mathematical details of this procedure (the form of the mean flow and of the perturbation, that of the linearised conditions, the resulting matrices) are given in the appendix.

These conditions lead to a $3 \times 3$ generalized eigenvalue problem $D X=c A X$, where the matrices $D$ and $A$ depend on the mean flow and on the physical parameters of the problem, and where the vector $X$ contains the coefficients of the perturbation streamfunction (see Appendix). This problem is solved numerically for our purposes. The eigenvalues $c$ are the solution of a cubic equation

$$
\left(c-C_{11}\right)\left(c-C_{12}\right)\left(c-C_{21}\right)=0
$$

The phase speeds $C_{11}, C_{12}, C_{21}$ correspond to Rossby waves propagating on the PV fronts; they depend, in a complex manner, on the PV jumps across the fronts, on the width of the PV cores, of the wavenumber, layer thicknesses and on the deformation radius. When any of the phase speeds is complex (in fact, when two of them become conjugate) for a given wavenumber $k$, the flow becomes unstable with growth rate $k \operatorname{Imag}(c)_{\max }$ and associated wavenumber $k$.

Linear barotropic and baroclinic instability can be analysed and explained both with instability criteria and from the point of view of Rossby wave resonance.

1. The Rayleigh-Kuo criterion for barotropic instability of parallel flows ([12]), and the Charney-Stern criterion for baroclinic instability $([4])$, state that the potential vorticity 
gradient of the mean flow must vanish and reverse, respectively on the horizontal, and in or between layers. Clearly, both criteria will be satisfied, whatever the sign of the PV cores. Indeed, the upper layer PV core has opposite-signed jumps at its two fronts, and one of these PV jumps will be of opposite sign to that in the lower layer, whatever $Q_{2} / Q_{1} \neq 0$.

2. Rossby wave resonance is the basis for these instabilities. These waves are due to the $\mathrm{PV}$ jumps across the fronts. Barotropic instability is favored when the waves in the same layer of the fluid are close to each other, that is, for narrow PV cores. Baroclinic instability is favored for cores of width comparable with the deformation radius. For like-signed PV cores, the baroclinic resonance occurs between the internal upper front and the lower front. But for opposite-signed PV cores, baroclinic instability involves the resonance of the lower PV front with the external upper PV front, a process which is clearly damped by too wide PV cores. To palliate this, longer waves, which can allow larger meridional displacements of the PV fronts, are needed.

Now we examine the maximum growth rates $k \operatorname{Imag}(c)_{\max }$ and the associated wavenumbers $k$ in the $\left(L_{1} / R d, Q_{2} / Q_{1}\right)$ parameter plane with $L_{2}=2 L_{1}$ (see figure 4 ). Clearly, the instability characteristics differ from like-signed to opposite-signed PV cores. When $Q_{1}$ and $Q_{2}$ are like-signed, both the growth rates and the most unstable wavenumbers decrease with increasing $L_{1} / R_{d}$. Barotropic instability is favored by narrow cores and is associated with short waves; baroclinic instability is favored by wide cores and is associated with long waves. But overall, barotropic instability is more efficient. When $Q_{1}$ and $Q_{2}$ have opposite signs, the variation in growth rate is not monotonic as $L_{1} / R_{d}$ increases from 0.5 to 2.0. After an initial decay, there is a secondary growth, e.g. for $L_{1} / R_{d} \geq 1.5$ when $Q_{2} / Q_{1} \sim-2.0$. Indeed, narrow cores favor barotropic instability. But here, baroclinic instability is most efficient for a finite width of the PV cores. Indeed, a narrow core has a small potential energy reservoir for a given stratification and thus baroclinic instability is damped. But for a very wide core, the lower PV front and the external upper layer PV front are far away, and the resonance of Rossby waves on these two fronts is damped. Therefore the maximal growth rates for baroclinic instability are found at finite $L_{1} / R_{d}$. For large $L_{1} / R_{d}$, long waves are necessary to couple the fronts. This is why the most unstable wavenumber is about 3 for $L_{1} / R_{d}=2, Q_{2} / Q_{1} \sim-3$ and about 1 for $L_{1} / R_{d}=2, Q_{2} / Q_{1} \sim 3$. Thus, for opposite-signed 
PV fronts, barotropic instability is favored for narrow PV cores. As the cores become wider, this type of instability is damped, and for a finite width of the cores, baroclinic instability becomes dominant.

Finally, for very wide PV cores, both types of instabilities are damped. Thus we should expect the existence of two families of unstable modes.

This is confirmed by figure 5 , which displays the growth rates of normal mode perturbations versus wavenumber for various values of $Q_{2} / Q_{1}$, when $L_{1} / R_{d}=1.5$. These plots confirm the existence of two families of unstable modes, each one associated to a relative maximum of growth rate, and corresponding respectively to short and to long waves, when the PV cores are opposite signed. It also shows that only one family of unstable modes exists when the PV cores are like-signed, which confirms our previous analysis. Finally, for oceanographic applications, we find that the growth periods are about $10-20$ days, and the unstable wavelengths of the order of 150 to $300 \mathrm{~km}$, which correspond to oceanic observations of the meanders on such currents.

\section{NONLINEAR REGIMES: CLASSIFICATION AND PHYSICAL MECHANISMS}

We performed experiments with a finite-difference code of the two-layer quasi-geostrophic equations. This numerical model and its initial conditions were described above. The values of the physical parameters, normalised core width $L_{1} / R_{d}$ and PV ratio $Q_{2} / Q_{1}$ were varied. The mean flow was initially perturbed by the most unstable wave computed in the previous section. In each case, the model was run for one year of simulated time, and we analysed the evolution of the PV field in the domain.

Firstly, one can recall the results of linear instability: for narrow PV cores, barotropic instability is expected to prevail, since this flow has strong horizontal velocity shears. On the contrary, for wide cores, baroclinic instability will dominate, if the layerwise PV's are like-signed. Indeed, in this case, the two PV fronts with opposite-signed PV jumps lie on top of each other. If the layerwise PV's are opposite-signed, the resonance of Rossby waves on the lower layer PV front with those on the external upper layer PV front will be rendered all the more difficult as the distance between these fronts increases.

The flow evolution displays different regimes depending on the relative values of the parameters $L_{1} / R_{d}$ and $Q_{2} / Q_{1}$ (see figure 6). Four main regimes (A,C,D,E) and three variants 
$(\mathrm{B}, \mathrm{F}, \mathrm{G})$ were observed:

Regime A is characterised by the breaking of short waves, leading to the formation of small vortices near the coast. This regime is called trapped instability at the coast. It occurs for both signs of $Q_{2} / Q_{1}$ and for narrow PV cores. Therefore, barotropic instability is expected to be favored, but baroclinic instability may be also strong when the layerwise PV's are like-signed. The strength of the instability leads to the irreversible evolution of the instability, manifested in physical space by wave breaking. The case of narrow PV cores also corresponds to the domain where short wavelengths are most unstable, hence the observed evolution.

Regime B is a variant of regime (A), where the like-signed PV cores align along the coast; this regime occurs for $Q_{2} / Q_{1}>0$ and for PV core widths comparable with the deformation radius; in this case, mixed barotropic-baroclinic instability is expected. This instability produces shoreward and offshore displacements of the PV cores. Since these cores are like-signed, the vertical alignment process ([14]; [8]) will take place and the cores will finally be superimposed.

Regime $\mathbf{C}$ corresponds to the formation of baroclinic dipoles near the coast and to their oscillatory evolution with the mirror images. This regime occurs for $Q_{2} / Q_{1}<0$, a necessary condition for the formation of baroclinic dipoles, and for PV core widths comparable with the deformation radius; it is expected that baroclinic instability will be most efficient then. In this case, a vertical phase shift of $\lambda / 4$ between meanders in the two layers will favor the coupling of these meanders to form baroclinic dipoles (here $\lambda$ is the wavelength of the perturbation). The closeness of the coast leads to a strong interaction of these dipoles with their mirror images.

Regime $\mathbf{D}$ is the formation and ejection of baroclinic dipoles; this regime occurs for $Q_{2} / Q_{1}<0$; the same remarks hold as for regime (C), but now, the upper layer vortices will be formed farther away from the coast. This will diminish the interaction of these vortices with their mirror images, thus allowing the ejection of the dipoles.

Regime $\mathbf{E}$ occurs when potential vorticity is present in the upper layer only. It shows the formation of a vortex street along the coast in this layer. In this case, barotropic 
instability acts alone. This evolution is the classical roll-up of a vorticity strip, slightly modified by stratification.

Regime $\mathbf{F}$ is a variant of regime (B) with a turbulent evolution near the coast. This regime occurs for $Q_{2} / Q_{1}>0$ and for wide PV cores. Since long waves are most unstable linearly in this case (see figure 4), they can interact nonlinearly to form multiple shorter waves, leading thus to a turbulent end-state (see also, e.g., [10]).

Regime $\mathbf{G}$ is a variant of regime (D) with the splitting of the lower layer vortices and the formation of baroclinic tripoles; this regime occurs for $Q_{2} / Q_{1}<0$ and also for wide PV cores (and thus for fairly large vortices).

Secondly, after relating the various regimes to the properties of linear instability, we can also state how, in the long run, the nonlinear effects may strongly modify the initial flow situation and properties.

In regimes $(\mathrm{A})$ and $(\mathrm{B})$, the vertical alignment of the PV cores will lead to the disappearance of the null vorticity core along the coast in the upper layer. As a consequence, the inner PV front of the upper layer will tend to disappear, a process which will damp barotropic instability.

In regimes $(\mathrm{C})$ and $(\mathrm{D})$, the formation of baroclinic dipoles completely disrupts the zonal mean flow. Regime $(\mathrm{C})$ is a (nearly) time-periodic state, while regime (D) corresponds to an irreversible evolution where the coastal waters are expelled offshore. Regime $(G)$ also possesses the character of irreversibility. Note that on very long time scales (on many oscillation periods), regime (C) finally destabilises and an evolution similar to that of regime (D) (dipole expulsion) is attained. This shows that a secondary instability, related to the oscillating state, and acting on much longer time-scales, is at work.

Finally, regime (F) also shows multiple stages in the instability where many harmonics successively grow via nonlinear interactions and modify the flow state. Contrary to regime (C), many harmonics grow so that no low-order system is established. 


\section{NONLINEAR REGIMES: INDIVIDUAL DESCRIPTION}

\subsection{Potential vorticity evolution and Fourier analysis of the perturbation}

For each regime, the time series of potential vorticity maps is shown, and Fourier analyses of the flow along the zonal axis complement the characterisation of the regimes. For each mode in $x$, the Fourier analysis provides meridionally varying amplitudes $A m p_{k}(y, t)$; the quadratic norm of these functions is computed for their $y$ dependence. This provides a time-dependent (only) amplitude $A_{k}(t)$ for each zonal mode $k$.

Figure 7 displays the time evolution of potential vorticity in the two layers for regime A with opposite signed potential vorticity in the two layers $\left(Q_{2} / Q_{1}=-1, L_{1} / R_{d}=0.6\right)$. At $t=50$, the breaking of the barotropically unstable short waves has produced a row of vortices in the upper layer. The lower layer PV front is perturbed by meanders. At $t=100$, vortices have clearly formed along the coast in the lower layer. At $t=150$ all vortices have grown to their final size and the configuration remains steady at $t=200$. The vortices do not pair vertically as baroclinic dipoles because (a) they are small and their area-integral of $\mathrm{PV}$ is weak, (b) the vertical coupling between layers is weak. Clearly here, the dominant mechanism is barotropic instability. We also notice that, though the layerwise vortices are opposite-signed here, they finally tend to align vertically. This is related to the dominance of horizontal interactions on vertical coupling here.

The time evolution of potential vorticity in regime A with like signed potential vorticity in the two layers, is shown in figure 8. The main difference with the previous regime is the absence of vortices in the lower layer, in the final stages, and the vertical alignment of the PV cores along the coast. This latter process is characteristic of like-signed PV anomalies.

The Fourier analysis of potential vorticity in the upper layer shows that, for about 300 days, the most unstable mode $k=10$ is dominant (see figure 9); but this mode grows only for $15-20$ days, and then stagnates and finally decreases. Its growth must be halted by nonlinear mechanisms. The observation of graver modes $(k=5,6)$ allow us to identify this mechanism. Indeed, these longer waves grow from small amplitude, both via linear instability, and by feeding on the energy of mode 10. Modes 4, 5, 6 and 10 attain the same amplitude only after a year of simulation. Thus, for the time shown in the simulation, mode 10 remains the strongest by far.

Such a long-term bifurcation towards longer waves is characteristic of the presence of 
multiple linearly unstable waves, the shorter ones being only slightly more unstable than the longer ones.

Regime $\mathrm{B}$ is shown in figure 10 . Longer waves are unstable here, and, at $t=50$, the upper layer core undergoes a classical roll-up into a vortex street, with little influence of the lower layer which displays only a few weak meanders. Again, this evolution indicates a dominant barotropic instability. Once vortices are formed in the upper layer, they remain attached to the coast and propagate eastward via mirror effect (see $t=100$ ). In the lower layer, the meanders shed filaments, which roll-up under the upper layer vortices. This roll-up amplifies the formation of identifiable, separate vortices in the lower layer (see $t=150,200$ ), which align vertically with the upper layer vortices.

Figure 11 illustrates this vertical alignment process by showing the meridional profile of the zonally averaged PV. Clearly, the two PV cores have widened due to the formation of vortices, but their maxima and their front are now aligned vertically.

Figure 12 shows the time evolution in regime C. Again, long waves are unstable, and the initial evolution of the flow (until $t=100$ ) is similar to that in the case of like-signed PV cores. Nevertheless, once the vortices have formed in both layers (at about $t=125$ ), they undergo two influences. Firstly they couple with their mirror image across the coast, and secondly they couple vertically.

The coupling with their mirror images advects them in opposite directions zonally (eastward for the upper layer vortices, westward for the lower layer ones). This advection brings upper and lower layer vortices closer to each other, by pairs, thereby increasing their vertical interaction. But since the horizontal interaction with the mirror images is stronger than the vertical one, the vortices do not leave the coast as hetons. They rotate around each other and then they continue drifting westward until they encounter with the following upper layer vortex in the row, and the whole process starts again. We call this periodic evolution an "oscillatory regime" because the layer wise vortices propagate zonally and oscillate meridionally periodically (when they are close to the opposite layer vortices). This regime will be studied in more details with point vortices in the following section.

The Fourier analysis of the upper layer potential vorticity (see figure 13) indicates that the linearly most unstable wave ("the fundamental", $k=4$ ) generates its first and second harmonics $(k=8,12)$ rapidly, while the other waves (initially weak) grow more slowly, and also interact nonlinearly (this is attested by the presence of many wavenumbers). The same 
waves $(k=4,8,12)$ emerge in the lower layer (not shown here). In the long-run, the fundamental remains of constant amplitude; this reflects the persistence of four distinct vortices. On the contrary, the first and second harmonics oscillate in relation with the periodic vortex evolution, also associated with the periodic production of smaller-scale filaments. The dominance of a small number of harmonics usually allows the simulation of the evolution via a low-order system of coupled ordinary differential equations, obtained by truncation of the full nonlinear system (e.g. [3]); but this is beyond the scope of the present paper.

In regime D (see figure 14), waves appear on all PV fronts, with a meridional phase tilt in the upper layer, and between the internal front in this layer and the lower front, indicating mixed barotropic-baroclinic instability. The roll-up of the upper layer PV core is now only slightly faster than that of the lower core, so that at $t=100$, opposite-signed vortices are formed in the two layers and pair baroclinically. This pairing of asymmetric vortices is accompanied by filamentation, and by curved trajectories of the baroclinic dipoles (or "hetons"). Finally, the vortices form a staggered vortex street with similar vortex sizes; this configuration attains a near steady state ( studied with point vortices. The Fourier analysis of regime D is similar to that of regime C. In both layers, the same waves grow.

Regime E corresponds to the case where the lower layer has zero potential vorticity. The nonlinear evolution corresponds to a rapid roll-up of the PV core into a symmetric vortex street (see figure 15).

Regime $\mathrm{F}$ differs from regime $\mathrm{B}$ in that several wavenumbers are linearly unstable. This leads to an asymmetric vortex street along the coast (see $t=150$ in figure 16). The vertical interaction between these vortices of different sizes leads to much filamentation and roll-up $(t=175$ and $t=225)$, and to a turbulent state along the coast $(t=250)$.

The Fourier analysis of the flow confirms that all waves with even wavenumbers have a much larger amplitude than those with odd wavenumbers. Indeed, the most unstable wave is $k=2$ and all its harmonics grow after a larger delay as $k$ increases. The irregular growth of waves beyond $k=4$ as well as the stabilisation of all waves at finite amplitude, is indicative of intense nonlinear interactions. Nevertheless, the multiplicity of wavenumbers finally involved in the perturbation renders a low-order model inoperative here.

Finally, regime $\mathrm{G}$ is a variant of regime $\mathrm{D}$ where vortices in the lower layer pair baroclinically (as hetons) with the upper layer vortices. But since the area-integral of potential 
vorticity in the upper layer vortices is large, it creates an intense shear on the lower layer vortices. The latter are strongly elongated and break into two parts. The association between each upper layer vortex and each pair of lower layer vortices forms a baroclinic tripole.

\subsection{Summary}

Essentially four regimes were obtained with variants:

1. $(\mathrm{A}, \mathrm{B}, \mathrm{F})$ trapped instability along the coast, either with the formation of a vortex street, of a current with meanders or of a turbulent flow. Regime A occurs for the most unstable waves which are short, and for both like-signed and opposite-signed PV cores. Regimes B and F occur for long waves and like-signed PV cores. In these cases, the baroclinic coupling of the PV anomalies in the two layers either leads to vertical alignment, or is not sufficient to create strong dipoles, which can leave the coast.

2. (C) The periodic oscillation of baroclinic vortices, under their mutual influence and under the influence of the mirror vortices. It occurs for intermediate-size waves and opposite-signed PV cores. In this case, the vortices interact predominantly with their mirror images across the wall.

3. (D,G) A dominant baroclinic instability with the formation of baroclinic dipoles (hetons) and of baroclinic tripoles, when the lower layer vortices split. These regimes dominate for long waves, and for opposite-signed PV cores. Then, the vertical coupling of opposite-signed vortices is strong enough to advect them away from the coast.

4. (E) the roll-up of the upper layer current into a vortex street as a consequence of pure barotropic instability. This regime is specific of upper-layer confined PV and of horizontal processes. Furthermore, it appears that barotropic instability dominates with short waves when the PV cores are narrow, and conversely baroclinic instability leads to long meanders on wide PV cores.

The Fourier analysis has shown that, for regime A, short waves emerge but due to linear growth and to nonlinear interactions, longer waves grow (these latter being about twice as long as the main wave). For regime $\mathrm{C}$ and $\mathrm{D}$, a main long wave grows to the largest amplitude, 
and feeds its first two harmonics. Finally, regime F is characterised by a multiplicity of (here, even) waves.

Applying these results to the Kuroshio, we have $Q_{2} / Q_{1} \sim-2, L_{1} / R_{d} \sim 1.5$ so that regime D would be favored. Nevertheless, beta effect has not been included up to here. We performed twenty more numerical simulations, now including beta effect in the nonlinear dynamics. The results are shown on figure 18. Comparing it with figure 6, one notices that like-signed PV cores evolve according to the same regimes as on the f-plane. This can be understood since beta effect tends to favor zonal flows, which are the end-state of the alignment process. The most important modification occurs for opposite signed PV cores for which regime D has vanished. Again, beta-induced zonalisation of the flow acts against the meridional ejection of vortices.

Therefore, on the beta-plane, we would expect the existence of eddies (or meanders) close to the coast, travelling in opposite directions in the two layers, for a current such as the Kuroshio. Altimetric sea-surface height maps show the westward propagation of anticyclonic eddies along the Kuroshio ([9]), but no data are available for the deeper flow, so that it is not presently possible to check if such a regime effectively occurs.

\section{STUDY OF THE DIPOLAR REGIMES WITH POINT VORTICES}

The purpose of the present section is to characterise the possible trajectories of the vortices, once they have detached from the coastal flow. In particular, we wish to discriminate the two regimes that correspond to the dipoles leaving the coast (D) and the dipole oscillation at the coast $(\mathrm{C})$. Indeed, we have seen that the Fourier analysis is not able to distinguish these two regimes. Therefore, we consider a stage where the unstable zonal currents have evolved into a double row of vortices that we model with point vortices. For simplicity we make several additional assumptions. Firstly, we assume that all the potential vorticity contained in an unstable wavelength $\lambda$ of the PV core has simply concentrated into one vortex, that is, the vortex strengths are

$$
\begin{aligned}
& \Gamma_{1}=Q_{1}\left(L_{2}-L_{1}\right) \lambda \\
& \Gamma_{2}=Q_{2} L_{1} \lambda
\end{aligned}
$$

For our applications, we have again chosen $L_{2}=2 L_{1}$ here. Note that $\lambda$ is given for each couple $\left(L_{1} / R_{d}, Q_{2} / Q_{1}\right)$. 
Secondly, we also assume that each vortex is located meridionally in the middle of its respective PV core, that is the vortices are located at distances $d_{1}=\left(L_{1}+L_{2}\right) / 2$ and $d_{2}=L_{1} / 2$ from the coast.

Finally, we assume that the vortices have moved zonally to concentrate into vortex pairs, and thus we neglect the interaction between vortex pairs themselves. For instance, for baroclinic instability, the vortices inside a pair would be separated by $\lambda / 4$ which corresponds to the maximum efficiency of baroclinic instability. Then, each pair would be separated from the following pair by $3 \lambda / 4$.

Vortex 1 has strength $\Gamma_{1}$ and is located in $x_{1}, y_{1}=d_{1}$, vortex 2 has strength $\Gamma_{2}$ and is located in $x_{2}, y_{2}=d_{2}$ and their mirror images have opposite strengths and are located in $x_{1},-y_{1}$ and $x_{2},-y_{2}$.

The Hamiltonian of the system is therefore

$$
H=\Gamma_{1} \psi\left(x_{1}, y_{1}\right)+\Gamma_{2} \psi\left(x_{2}, y_{2}\right)
$$

where

$$
\begin{aligned}
& \psi\left(x_{1}, y_{1}\right)=\Gamma_{2} G_{21}\left(r_{12}\right)-\Gamma_{2} G_{21}\left(r_{1-2}\right)-\Gamma_{1} G_{11}\left(r_{1-1}\right) \\
& \psi\left(x_{2}, y_{2}\right)=\Gamma_{1} G_{12}\left(r_{12}\right)-\Gamma_{2} G_{22}\left(r_{2-2}\right)-\Gamma_{1} G_{12}\left(r_{2-1}\right)
\end{aligned}
$$

The $G_{i j}$ are the Green's functions between layers $i$ and $j$ which are written

$$
\begin{aligned}
& G_{11}(r)=h_{1} G^{0}(r)-h_{2} G^{1}(r) \\
& G_{12}(r)=h_{2} G^{0}(r)+h_{2} G^{1}(r) \\
& G_{21}(r)=h_{1} G^{0}(r)+h_{1} G^{1}(r) \\
& G_{22}(r)=h_{2} G^{0}(r)-h_{1} G^{1}(r)
\end{aligned}
$$

with $G^{0}(r)=\frac{1}{2 \pi} \ln (r), G^{1}(r)=-\frac{1}{2 \pi} K_{0}(\gamma r), K_{0}$ the modified Bessel function of second kind of order zero, and $\gamma=1 / R_{d}$. The distances $r_{i j}$ between the point vortices are

$$
\begin{aligned}
r_{12}^{2}=r_{21}^{2} & =\left(x_{1}-x_{2}\right)^{2}+\left(y_{1}-y_{2}\right)^{2} \\
r_{1-2}^{2}=r_{2-1}^{2} & =\left(x_{1}-x_{2}\right)^{2}+\left(y_{1}+y_{2}\right)^{2} \\
r_{1-1} & =2 y_{1} \\
r_{2-2} & =2 y_{2}
\end{aligned}
$$


Figure 19 shows the isolines of the Hamiltonian in the $\left(x_{2}-x_{1}, L_{1} / R_{d}\right)$ plane, for $Q_{2} / Q_{1}=$ $\pm 2 . x_{2}-x_{1}$ is the zonal distance between the two vortices inside a pair, $L_{1} / R_{d}$ is the normalised width of the PV cores, which is related to the meridional position of the vortices.

For positive $Q_{2} / Q_{1}$, the isolines of $H$ show that two vortices, initially distant from each other (large values of $x_{2}-x_{1}$ ) can come close to each other and to the coast; this corresponds to regime $\mathrm{B}$ where like-signed vortices can align vertically and come close to the coast.

For negative $Q_{2} / Q_{1}$, the isolines of $H$ show two separate domains:

1. for vortices close to each other and to the coast, the isolines are curved in a bounded domain in $L_{1} / R_{d}$ when $x_{2}-x_{1}$ is small. This corresponds to motions where the vortices can rotate around each other when they come in close vicinity. This is the case for $L_{1} / R_{d} \sim 1$, where we observed regime C.

2. for vortices more distant from the coast, the isolines of $H$ are deflected towards larger values of $L_{1} / R_{d}$ as $x_{2}-x_{1}$ decreases. As $L_{1}$ increases, so do $d_{1}$ and $d_{2}$. Thus, as two vortices move towards each other (as they pair to form a baroclinic dipole), the two vortices tend to move away from the coast. This corresponds to regime D.

In a second stage, we could consider the Hamiltonian for a baroclinic, staggered, von Kàrman street, in relation with regimes (C) and (D) which form double rows of vortices along the coast. Most of the calculations are detailed in [13], and in [11], so that they are not repeated here. In short, we use Gryanik's results that we restrict to the $f$-plane. We also assume that $h_{1}=h_{2}=1 / 2$.

The Hamiltonian of the regular von Kármán street shows few differences between regimes B, C and D, contrary to the four vortex model. This is due in particular to the absence of isolation of vortex dipoles in this model. Indeed each vortex in a zonal row of the street is equally distant to his left and right neighbours in the other row, and thus their mutual influences on shoreward or offshore advection, cancel out.

\section{SUMMARY, CONCLUSION}

The linear stability and the nonlinear evolution of a coastal current composed of two cores of uniform potential vorticity, vertically shifted, were studied in a two-layer quasigeostrophic model. The cases of like-signed and of opposite-signed cores were addressed. 
The co-existence of barotropic and baroclinic instabilities is a novelty of the present study. Indeed, this flow configuration, though idealised, contains an ingredient observed at sea but never studied specifically: the shift between two cores of PV in the two different layers of water. Such a configuration may be relevant for the Kuroshio off the coast of Japan and also for the Mediterranean Water undercurrents off Portugal.

The linear stability analysis indicates that, for narrow vorticity cores, short waves dominate, associated with barotropic instability; for wider cores, longer waves are more unstable and are associated with baroclinic processes. The transition between these families of modes is smooth for like-signed cores and discontinuous for opposite-signed cores. The linear instability characteristics were interpreted in terms of the resonance of Rossby waves on the PV fronts.

Numerical simulations of the nonlinear evolutions of the coastal current were then performed with a two-layer quasi-geostrophic finite-difference code in a zonal channel configuration, firstly on the f-plane. These experiments showed that, when both cores have like-signed PV, trapped instability develops during the nonlinear evolution: vertical alignment of the PV cores is observed. For narrow cores, short wave breaking occurs close to the coast; for wider cores, substantial turbulence results from the entrainment of ambient fluid into the coastal jet.

When the two cores have opposite-signed PV, the nonlinear regimes range from short wave breaking to the ejection of dipoles or tripoles, via a regime of dipole oscillation near the wall.

These regimes were explained in terms of the properties of linear instability, but also in terms of interactions of PV anomalies, either for quasi-zonal flows along the coast, or once the meanders of the layerwise currents have occluded as vortices. In particular, three important aspects must be underlined:

1. the vertical alignment of the like-signed PV cores along the coast is the manifestation of a well-known process, but it has here the supplementary effect of closing off the source for barotropic instability;

2. there is a competition between inter-layer vortex interaction, which results in the ejection of baroclinic dipoles, and intra-layer vortex interactions, whereby each vortex couples with its mirror image and propagates along the coast; 
3. for large enough detached vortices, the inter-layer interaction can result in vortex breaking; this is due to the shear exerted by the upper on the lower layer vortices.

Therefore, the efficiency of the instability in exporting coastal waters offshore and in mixing it there, depends on a sequence of nonlinear processes, starting with the instability of the coastal current itself.

The addition of beta effect acts against the ejection of baroclinic dipoles and, as expected, tends to stabilise the flow in a zonal configuration. An application of these model results to the Kuroshio indicates that eddies can form and travel along the coast, a situation a priori observed.

The Fourier analysis of the perturbed flow was able to distinguish the regimes of short wave breaking, of dipole formation, and of turbulence, but not the differences between regimes involving only vortex pairs. To explain more precisely the regimes where two vortices (and their wall images) interact, a point vortex model is appropriate. This model was successful when only two vortices were considered, with their wall images. This situation accounts for the isolation of vortex pairs from their neighbours in the last stage of the instability. On the contrary, a regular point vortex street was not appropriate to distinguish the nonlinear evolutions identified in the numerical simulations.

To add realism to this configuration, bottom topography should be added; its expected influence would be towards the trapping of the lower PV core near the coast. A finer stratification would allow the representation of smaller-scale features in the horizontal plane, and vertical splitting effects of each PV core, which are not possible in our model. Nevertheless, an on-going study shows that the basic mechanism of PV front interaction is similar in a four-layer configuration.

\section{APPENDIX: MATHEMATICAL DETAILS OF THE LINEAR STABILITY}

\section{ANALYSIS}

Here we provide the form of the velocity profile for the mean flow; we detail the kinematic and dynamical conditions bearing on the perturbation, and we provide the form of the linear stability matrix $G$. 
Firstly the mean flow is computed by inverting the unperturbed potential vorticity distribution. This is achieved by projecting the layerwise components onto vertical modes via the formulae:

$$
X_{t}=h_{1} X_{1}+h_{2} X_{2}, \quad X_{c}=X_{1}-X_{2}
$$

where $h_{j}=H_{j} /\left(H_{1}+H_{2}\right), \quad j=1,2$, with subscripts $(1,2)$ for the upper and lower layers respectively. The subscripts "t" and "c" denote the barotropic and baroclinic modes respectively. The inverse formula is

$$
X_{1}=X_{t}+h_{2} X_{c}, \quad X_{2}=X_{t}-h_{1} X_{c}
$$

Using this, we have

$$
\bar{q}_{t}(y)=-\frac{d \bar{U}_{t}}{d y}, \quad \bar{q}_{c}(y)=\frac{d^{2} \bar{\psi}_{c}}{d y^{2}}-\frac{\bar{\psi}_{c}}{R_{d}^{2}}
$$

and we apply the boundary conditions that $\bar{U}_{t}$ must vanish at infinity in $y$ and be continuous at each potential vorticity jump $\left(y=L_{1}, y=L_{2}\right)$, and that $\bar{\psi}_{c}$ must vanish at the origin $(y=0)$ and at infinity in $y$ and must also be continuous at each potential vorticity jump.

Thus we have

$$
\begin{gathered}
\bar{U}_{t}(y)=h_{2} Q_{2}\left(L_{1}-y\right)+h_{1} Q_{1}\left(L_{2}-L_{1}\right), \quad 0 \leq y \leq L_{1} \\
\bar{U}_{t}(y)=h_{1} Q_{1}\left(L_{2}-y\right), \quad L_{1} \leq y \leq L_{2} \\
\bar{U}_{t}(y)=0, \quad L_{2} \leq y \\
\bar{\psi}_{c}=Q_{2} R_{d}^{2}+A_{c}^{a} e^{y / R_{d}}+B_{c}^{a} e^{-y / R_{d}}, \quad 0 \leq y \leq L_{1} \\
\bar{\psi}_{c}=-Q_{1} R_{d}^{2}+A_{c}^{b} e^{y / R_{d}}+B_{c}^{b} e^{-y / R_{d}}, \quad L_{1} \leq y \leq L_{2} \\
\bar{\psi}_{c}=B_{c}^{c} e^{-y / R_{d}}, \quad L_{2} \leq y
\end{gathered}
$$

with the following relations

$$
\begin{gathered}
A_{c}^{b}=\frac{Q_{1}}{2} R_{d}^{2} e^{-L_{2} / R_{d}}, B_{c}^{a}=-Q_{2} R_{d}^{2}+\frac{Q_{1}+Q_{2}}{2} R_{d}^{2} e^{-L_{1} / R_{d}}-\frac{Q_{1}}{2} e^{-L_{1} / R_{d}} \\
A_{c}^{a}=-Q_{2} R_{d}^{2}-B_{c}^{a}, B_{c}^{b}=B_{c}^{a}+\frac{Q_{1}+Q_{2}}{2} R_{d}^{2} e^{L_{1} / R_{d}}, B_{c}^{c}=B_{c}^{b}-\frac{Q_{1}}{2} R_{d}^{2} e^{L_{2} / R_{d}} .
\end{gathered}
$$

Then we write the perturbation streamfunction in vertical modes for each region of the flow by enforcing the condition that the perturbation has zero potential vorticity

$$
\psi_{t}^{\prime}=\alpha_{t}^{a} e^{k y}+\beta_{t}^{a} e^{-k y}, \quad 0 \leq y \leq L_{1}
$$




$$
\begin{gathered}
\psi_{t}^{\prime}=\alpha_{t}^{b} e^{k y}+\beta_{t}^{b} e^{-k y}, \quad L_{1} \leq y \leq L_{2} \\
\psi_{t}^{\prime}=\beta_{t}^{c} e^{-k y}, \quad L_{2} \leq y \\
\psi_{c}^{\prime}=\alpha_{c}^{a} e^{K_{\gamma} y}+\beta_{c}^{a} e^{-K_{\gamma} y}, \quad 0 \leq y \leq L_{1} \\
\psi_{c}^{\prime}=\alpha_{c}^{b} e^{K_{\gamma} y}+\beta_{c}^{b} e^{-K_{\gamma} y}, \quad L_{1} \leq y \leq L_{2} \\
\psi_{c}^{\prime}=\beta_{c}^{c} e^{-K_{\gamma} y}, \quad L_{2} \leq y
\end{gathered}
$$

where $K_{\gamma}^{2}=k^{2}+1 / R_{d}^{2}$. Thus we have ten unknowns for the linear instability problem.

Firstly, two relations are obtained by imposing that the meridional perturbation velocity must vanish at $y=0$.

$$
\alpha_{t}^{a}+\beta_{t}^{a}=0, \quad \alpha_{c}^{a}+\beta_{c}^{a}=0
$$

Secondly, the continuity of the perturbation streamfunction at the PV jumps leads to four relations

$$
\begin{gathered}
2 \alpha_{t}^{a} \sinh \left(k L_{1}\right)=\alpha_{t}^{b} e^{k L_{1}}+\beta_{t}^{b} e^{-k L_{1}} \\
2 \alpha_{t}^{a} \sinh \left(K_{\gamma} L_{1}\right)=\alpha_{t}^{b} e^{K_{\gamma} L_{1}}+\beta_{t}^{b} e^{-K_{\gamma} L_{1}} \\
\beta_{t}^{c}=\beta_{t}^{b}+\alpha_{t}^{b} e^{2 k L_{2}} \\
\beta_{c}^{c}=\beta_{c}^{b}+\alpha_{c}^{b} e^{2 K_{\gamma} L_{2}}
\end{gathered}
$$

Thirdly, $u_{2}^{\prime}$ is continuous in $y=L_{2}$ (in the absence of any PV jump there); thus, using previous relations, we obtain a supplementary relation

$$
k e^{k L_{2}} \alpha_{t}^{b}=h_{1} K_{\gamma} e^{K_{\gamma} L_{2}} \alpha_{c}^{b}
$$

Therefore, we are left with only three unknowns.

Finally, we implement the linearised potential vorticity equation around each PV front as

$$
\left(U_{j}\left(L_{k}\right)-c\right)\left[u_{j}^{\prime}\right]_{L_{k}-\epsilon}^{L_{k}+\epsilon}=\psi_{j}^{\prime}\left(L_{k}\right)\left[\bar{q}_{j}\right]_{L_{k}-\epsilon}^{L_{k}+\epsilon}
$$

where $[X]_{a}^{b}$ denotes the jump of the quantity $X$ between $a$ and $b$, and we let $\epsilon$ tend to zero. This provides three equations for the remaining three unknowns, after elimination of the previous seven unknowns using the seven relations above. These three equations involve $c$ which contains the phase speed and the growth rate of the perturbation. 
Setting $U_{1}=\bar{U}_{1}\left(L_{1}\right), U_{2}=\bar{U}_{1}\left(L_{2}\right), U_{3}=\bar{U}_{2}\left(L_{1}\right), \lambda_{t}=k / \sinh \left(k L_{1}\right), \lambda_{c}=K_{\gamma} / \sinh \left(K_{\gamma} L_{1}\right)$, $\mu_{t}=-2 k e^{k L_{2}}, \mu_{c}=-2 K_{\gamma} e^{K_{\gamma} L_{2}}$, we write the following terms of $3 \mathrm{x} 3$ matrices

$$
\begin{gathered}
a_{11}=\lambda_{t}+\frac{h_{2}}{h_{1}} \frac{\mu_{t}}{\mu_{c}} \lambda_{c}, a_{12}=\lambda_{t}, a_{13}=h_{2} \lambda_{c} \\
a_{21}=\frac{\mu_{t}}{h_{1}}, a_{22}=0, a_{23}=0 \\
a_{31}=-\lambda_{t}+\lambda_{c} \frac{\mu_{t}}{\mu_{c}}, a_{32}=-\lambda_{t}, a_{33}=h_{1} \lambda_{c}
\end{gathered}
$$

and

$$
\begin{aligned}
& b_{11}=Q_{1}\left(e^{k L_{1}}+\frac{h_{2}}{h_{1}} \frac{\mu_{t}}{\mu_{c}} e^{K_{\gamma} L_{1}}\right), b_{12}=Q_{1} e^{-k L_{1}}, b_{13}=h_{2} Q_{1} e^{-K_{\gamma} L_{1}} \\
& b_{21}=Q_{1}\left(e^{k L_{2}}+\frac{h_{2}}{h_{1}} \frac{\mu_{t}}{\mu_{c}} e^{K_{\gamma} L_{2}}\right), b_{22}=Q_{1} e^{-k L_{2}}, b_{13}=h_{2} Q_{1} e^{-K_{\gamma} L_{2}} \\
& b_{31}=Q_{2}\left(e^{k L_{1}}-\frac{\mu_{t}}{\mu_{c}} e^{K_{\gamma} L_{1}}\right), b_{32}=Q_{2} e^{-k L_{1}}, b_{33}=-Q_{2} h_{1} e^{-K_{\gamma} L_{1}}
\end{aligned}
$$

Finally, we defined $d_{j k}=U_{j} a_{j k}-b_{j k}$ so that $C_{11}, C_{12}, C_{21}$ (mentioned in section 3 in the main text) are the values of $c$ in the generalised eigenvalue/eigenvector problem

$$
d_{j k} X_{k}=c a_{j k} X_{k}
$$

We chose to solve this problem numerically, for our purposes, and we varied the physical parameters of the problem.

This problem could be solved analytically, but to the expense of long calculations. Define matrix $D\left(d_{j k}\right)$, matrix $A\left(a_{j k}\right)$ and matrix $G$ as the product $G=A^{-1} D$. The eigenvalue problem is then $G X=c X$, and the cubic equation in $c$ mentioned in the main text is obtained by canceling the $3 \times 3$ determinant of $G-c I$ where $I$ is the identity matrix. We do not provide an explicit expression of $C_{11}, C_{12}, C_{21}$ in terms of the parameters of the problem due to the complexity of the formulae. These three values are the (complex) Rossby phase speeds associated to the three PV jumps (this can be shown simply in the case of a single $\mathrm{PV}$ jump where the phase speed is real). 


\section{ACKNOWLEDGMENTS}

This work was made possible by a contract from the French National Agency for Research, under the STATOCEAN project of the SYSCOMM program.

1. X. Capet and X. Carton. Nonlinear regimes of baroclinic boundary currents. J. Phys. Oceanogr., 34:1400-1409, 2004.

2. X. Capet, L. Chérubin, and Y. Morel. Influence of transport on the instability of a boundary current. J. Phys. Oceanogr., 32:2806-2815, 2002.

3. X. Carton and J. Mc Williams. Barotropic and baroclinic instabilities of axisymmetric vortices in a qg model. In Mesoscale/synoptic coherent structures in geophysical turbulence, volume 50, pages 225-244. Elsevier Oceanographic Series, 1989.

4. J. Charney and Stern M. On the instability of internal baroclinic jets in a rotating atmosphere. J. Atmos. Sci., 19:159-172, 1962.

5. L. Chérubin. Descriptive analysis of the hydrology and currents on the iberian shelf from gibraltar to cape finisterre, preliminary results of the interafos and semane experiments. Annales. Hydrog., 21,768:5-70, 1997.

6. L. Chérubin, Y. Morel X. Carton, J. Paillet, and A. Serpette. Instability of the mediterranean water undercurrents southwest of portugal: Effect of baroclinicity and of topography. Oceanologica Acta, 23,5:551-573, 2000.

7. L. Chérubin, X. Carton, and D. Dritschel. Vortex dipole formation by baroclinic instability of boundary currents. J. Phys. Oceanogr., 37:1661-1677, 2007.

8. S. Corréard and X. Carton. Vertical alignment of geostrophic vortices: On the influence of the initial distribution of potential vorticity. In Simulation and identification of organized structures in flows, volume 52, pages 191-200. Kluwer Acad. Publ., 1998.

9. N. Ebuchi and K. Hanawa. Influence of mesoscale eddies on variations of the kuroshio path south of japan. J. Oceanogr., 59:25-36, 2003.

10. G. Flierl, X. Carton, and C. Messager. Vortex formation by unstable oceanic jets. In European Series in Applied and Industrial Mathematics, volume 7, pages 137-150. SMAI, Paris, 1999.

11. V. Gryanik, H. Borth, and D. Olbers. The theory of quasi-geostrophic von kármán vortex 
streets in two-layer fluids on a beta-plane. J. Fluid Mech, 505:23-57, 2004.

12. H. Kuo. Dynamic instability of two-dimensional nondivergent flow in a barotropic atmosphere. J. Meteor., 6:105-122, 1949.

13. A. Masuda and K. Miki. On the stability of baroclinic vortex streets composed of quasigeostrophic point eddies. Deep Sea Res., 42:437-453, 1995.

14. L. Polvani. Two-layer geostrophic vortex dynamics part 2. alignment and two-layer v-states. J. Fluid Mech., 225:241-270, 1991.

15. B. Qiu and W. Miao. Kuroshio path variations south of japan: Bimodality as a self-sustained internal oscillation. J. Phys. Oceanogr., 30:2124-2137, 2000.

16. K. Wyrtki, L. Magaard, and J. Hager. Eddy energy in the oceans. J. Geophys. Res., 81: 2641-2646, 1976. 

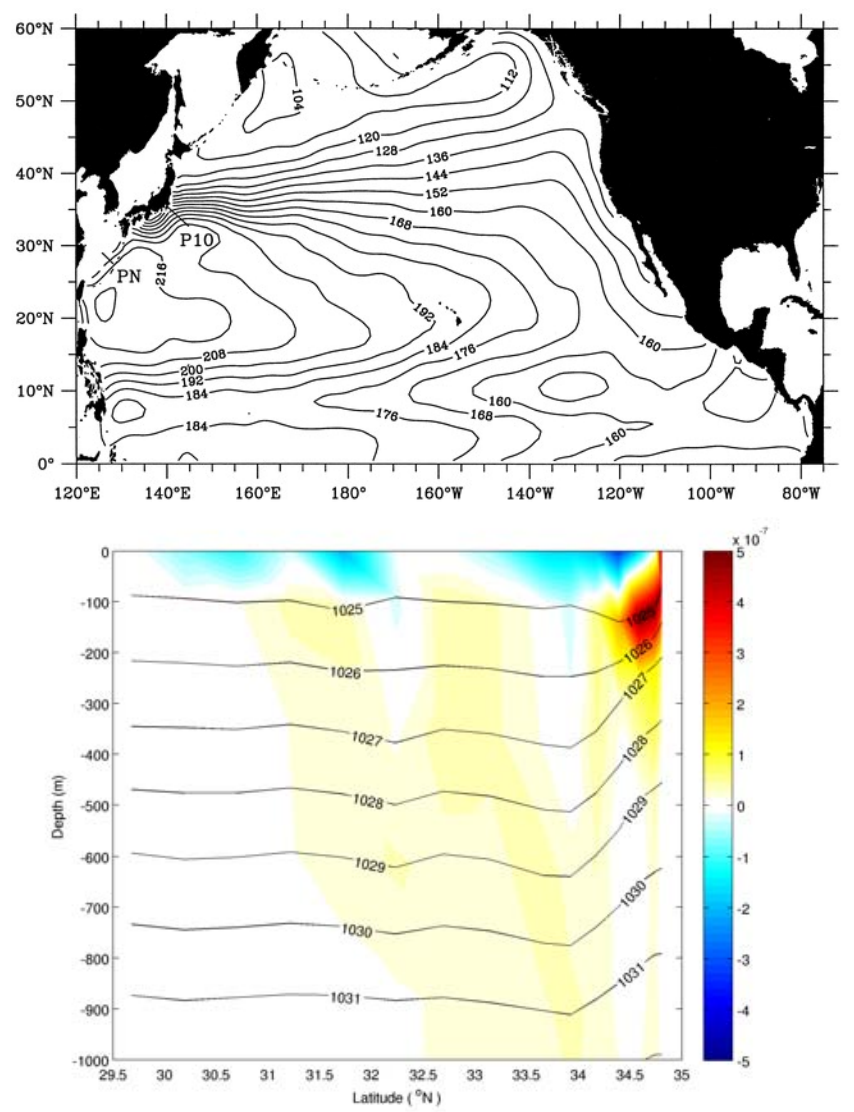

Figure 1. (Top) Mean sea surface dynamic topography relative to 1000 dbar, in centimeters. Line P10 denotes the CTD and lowered ADCP section of the WOCE P10 cruise. Adapted from [15].

(Bottom) Vertical section of the PV anomalies of the Kuroshio along P10 - the coast of Japan is on the right of the picture.

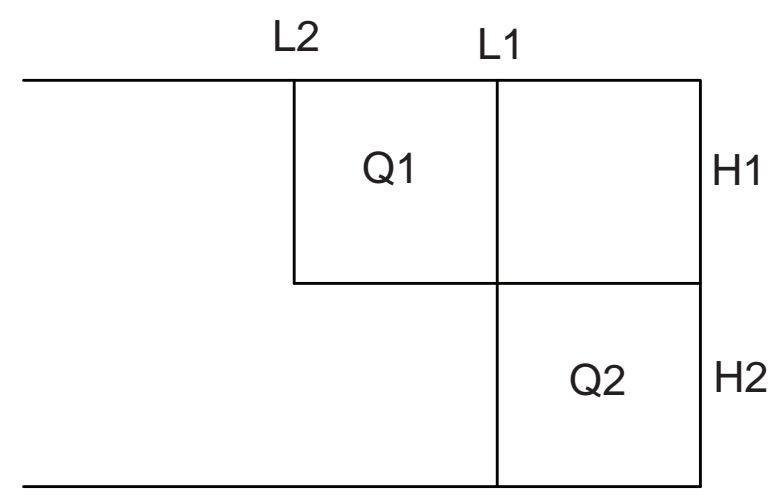

Figure 2. Two core structure of the potential vorticity distribution; the coast is on the right. 

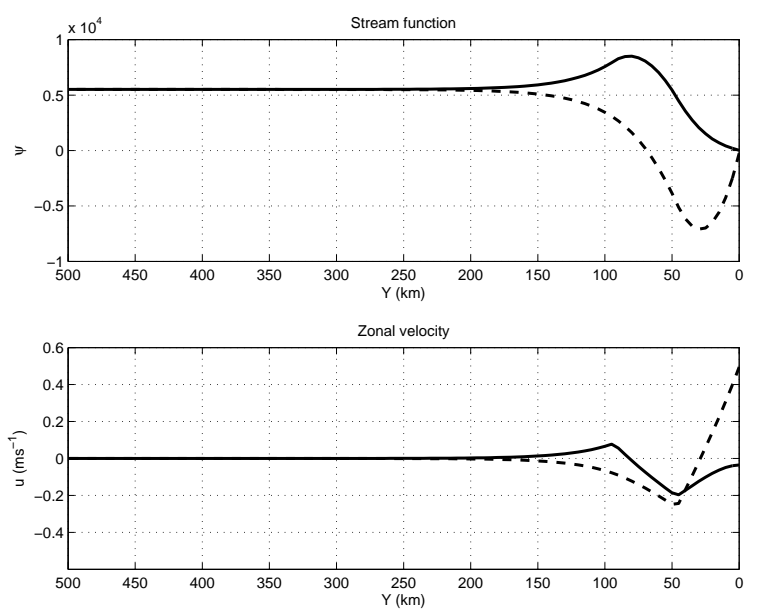

Figure 3. Meridional profile of the mean flow streamfunction (above) and velocity(below). Solid line corresponds to the upper layer and the dashed line to the lower layer.

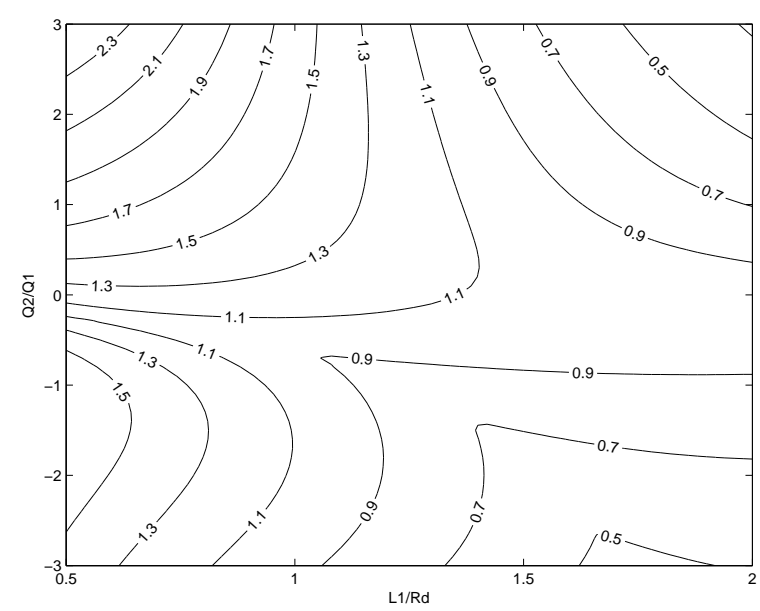

(a)

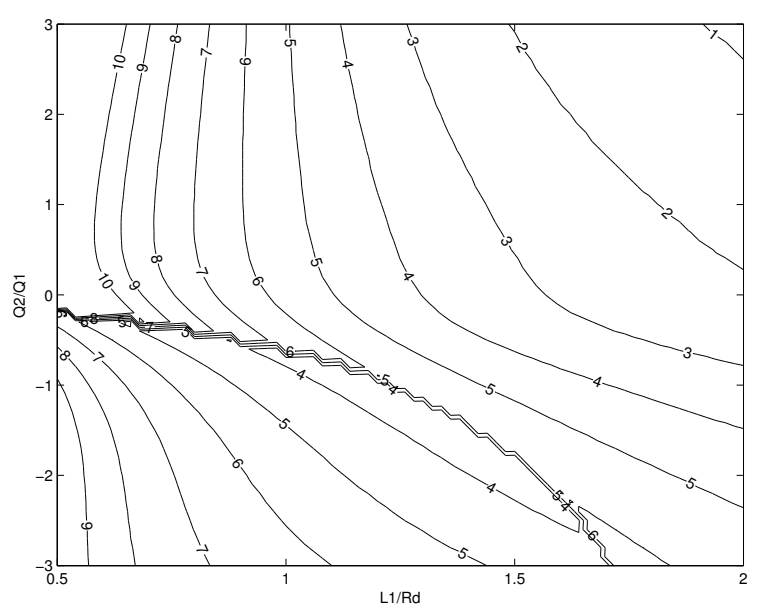

(b)

Figure 4. (a) Maximum growth rate as a function of the lower to upper layer PV ratio $Q_{2} / Q_{1}$ and of the normalised width of the upper layer PV core $L 1 / R_{d}$; (b) most unstable wavenumber $k$ in the same parameter plane. 

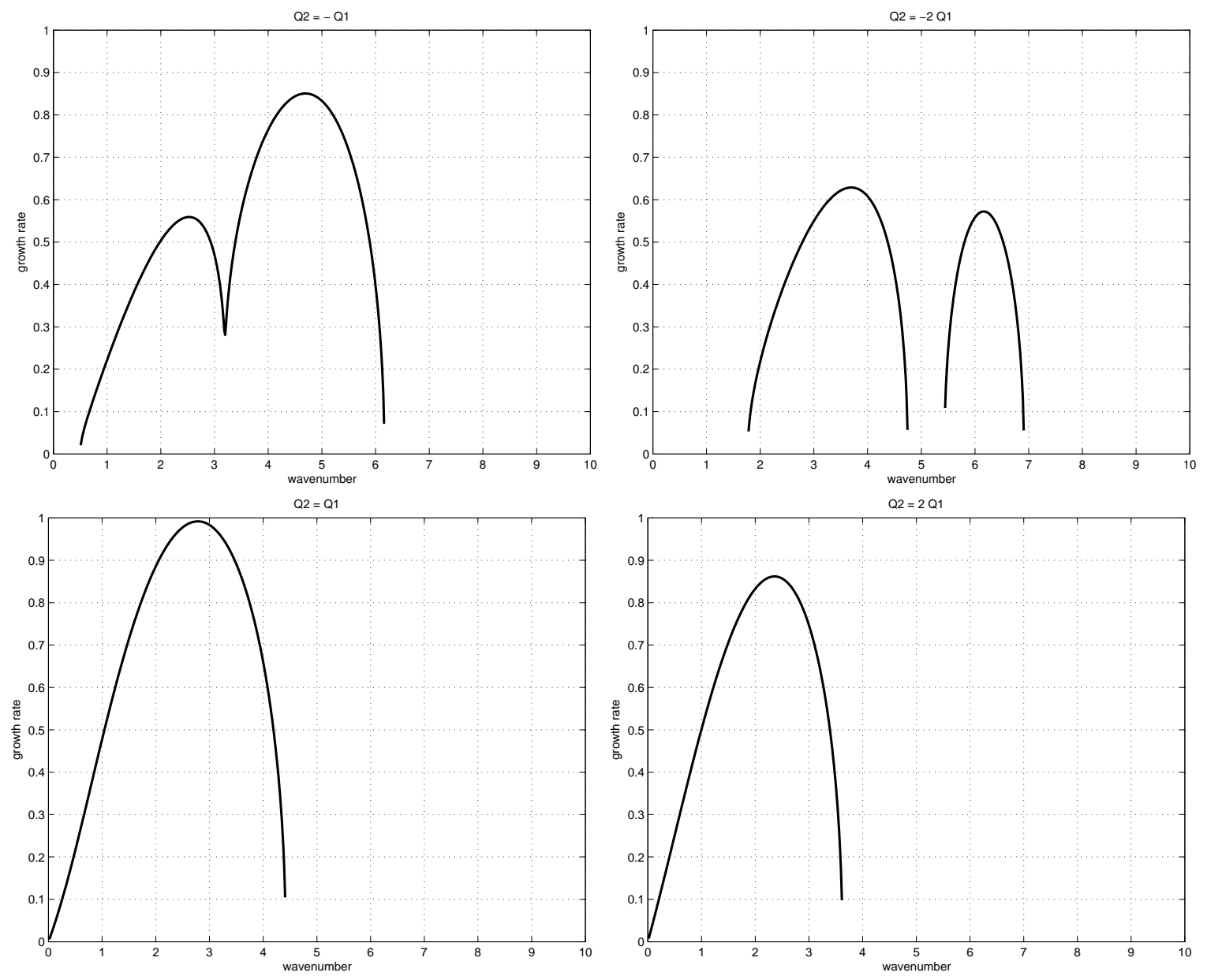

Figure 5. Growth rates of the normal modes versus wavenumber for several values of $Q_{2} / Q_{1}$ and

$$
L 1 / R_{d}=1.5
$$

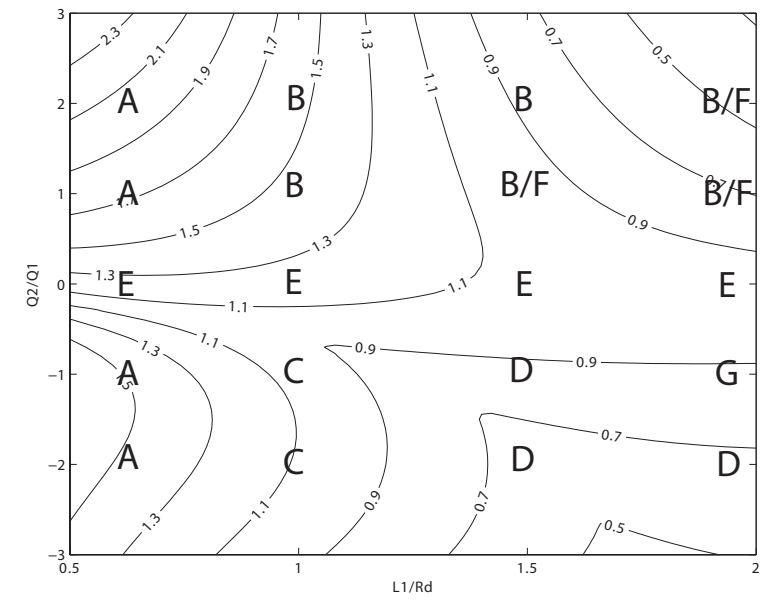

Figure 6. Classification of nonlinear regimes of flow in parameter space, superimposed on the growth rates of normal mode perturbations (thin lines, see also figure 4a). 

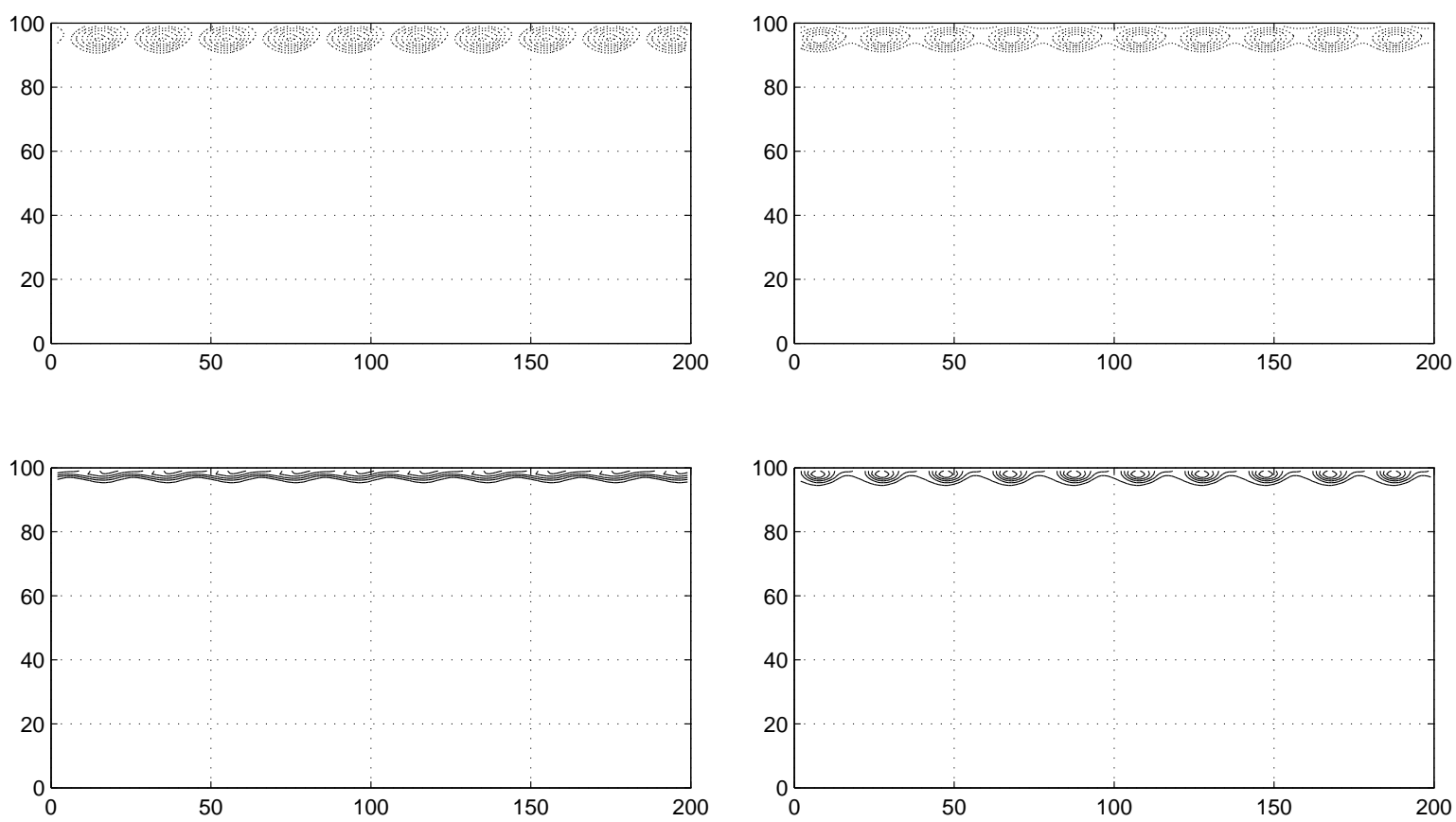

(a) $t=50$

(b) $t=100$
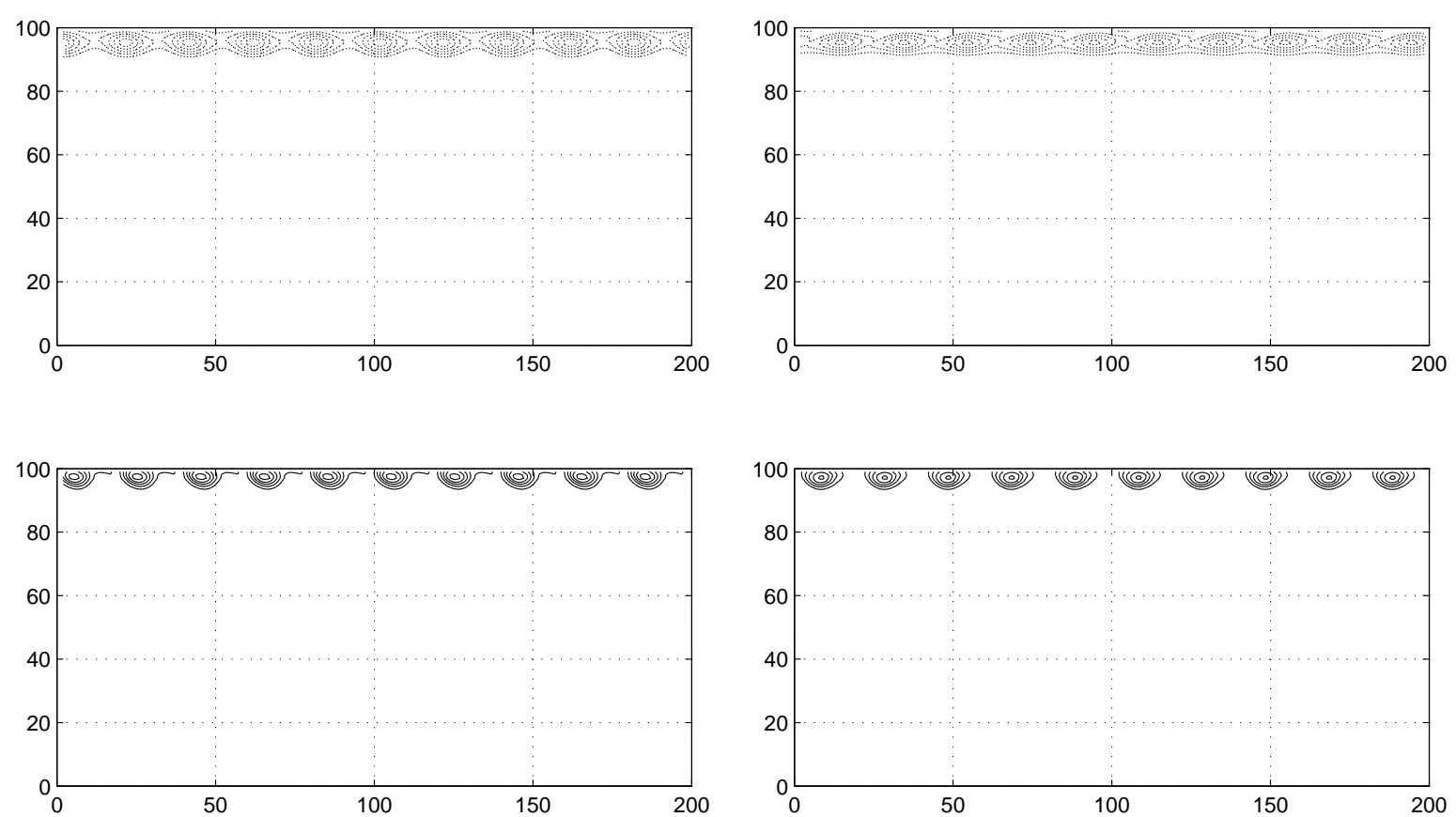

(c) $t=150$

(d) $t=200$

Figure 7. Non-linear evolution of the potential vorticity in the upper layer (above) and in the lower layer (below) for regime A with $Q_{2} / Q_{1}=-1$ and $L_{1} / R_{d}=0.6$. Positive/negative values of PV are represented by solid/dashed lines. From this figure, to figure 13, the computational domain is measured with grid numbers; these numbers must be multiplied by $5 \mathrm{~km}$ to give physical dimensions (length, width). 

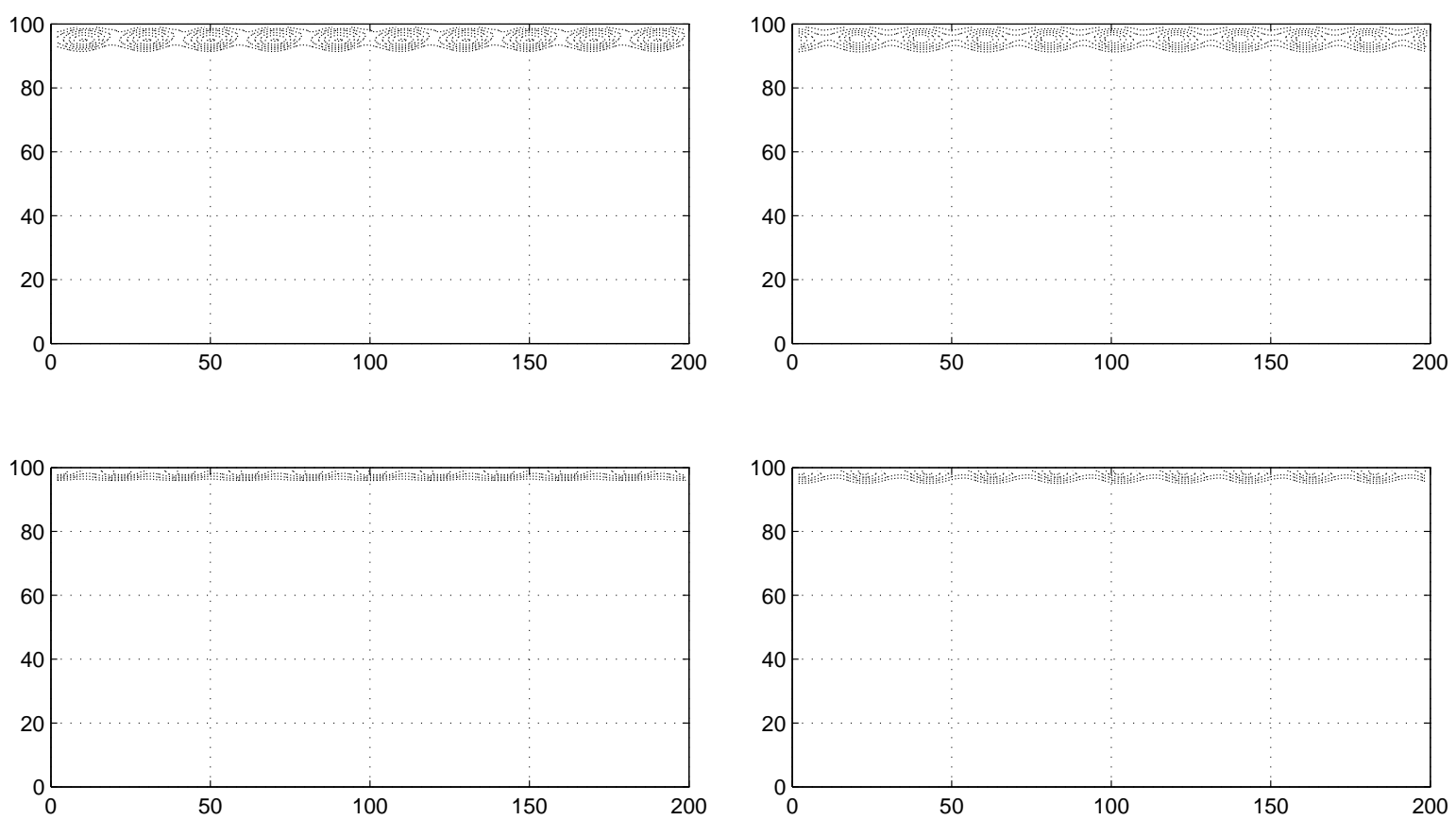

(a) $t=50$

(b) $t=100$
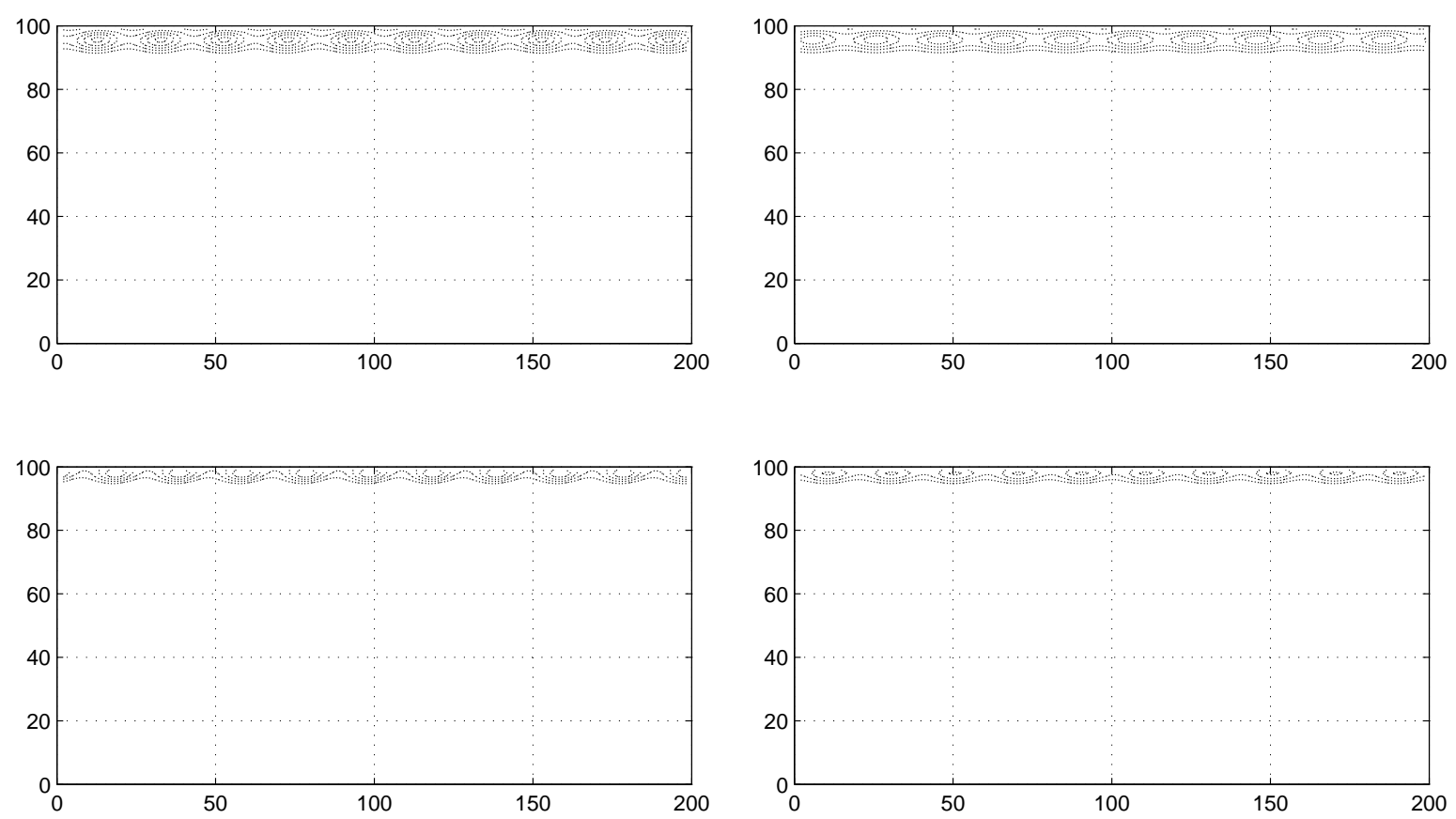

(c) $t=150$

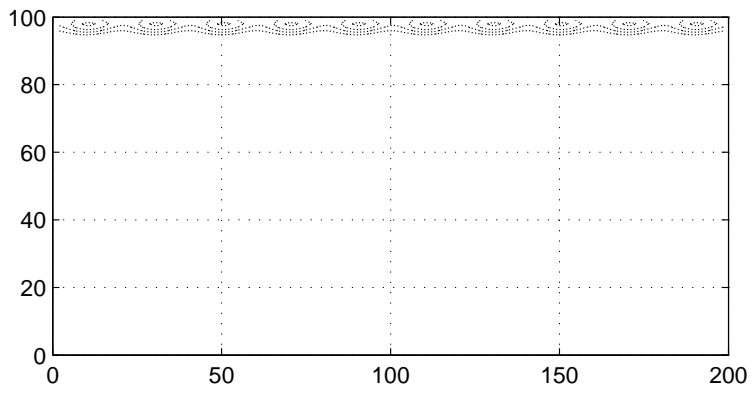

(d) $t=200$

Figure 8. Non-linear evolution of the potential vorticity in the upper layer (above) and in the lower layer (below) for regime A with $Q_{2} / Q_{1}=1$ and $L_{1} / R_{d}=0.6$. Positive/negative values of PV are represented by solid/dashed lines. 


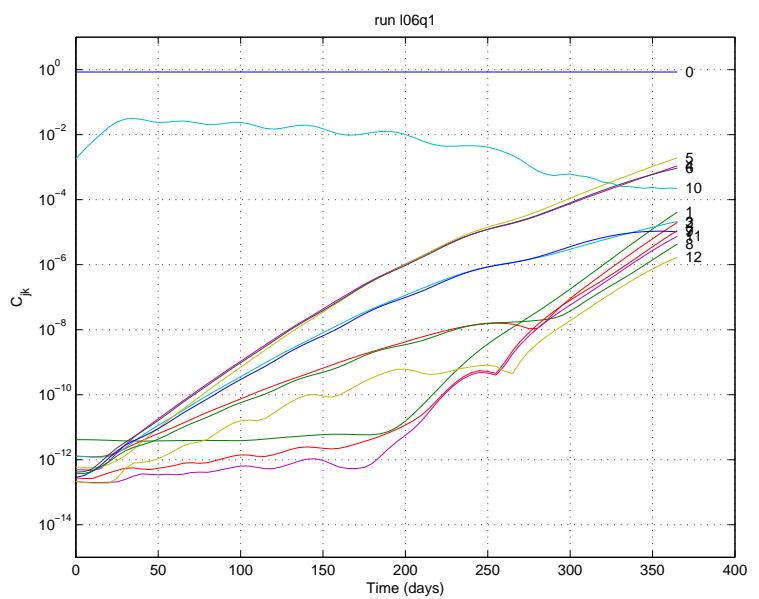

Figure 9. Fourier analysis along the zonal axis, of potential vorticity in the upper layer for regime A with $Q_{2} / Q_{1}=1$ and $L_{1} / R_{d}=0.6$; the numbers next to each line are the wavenumbers. 

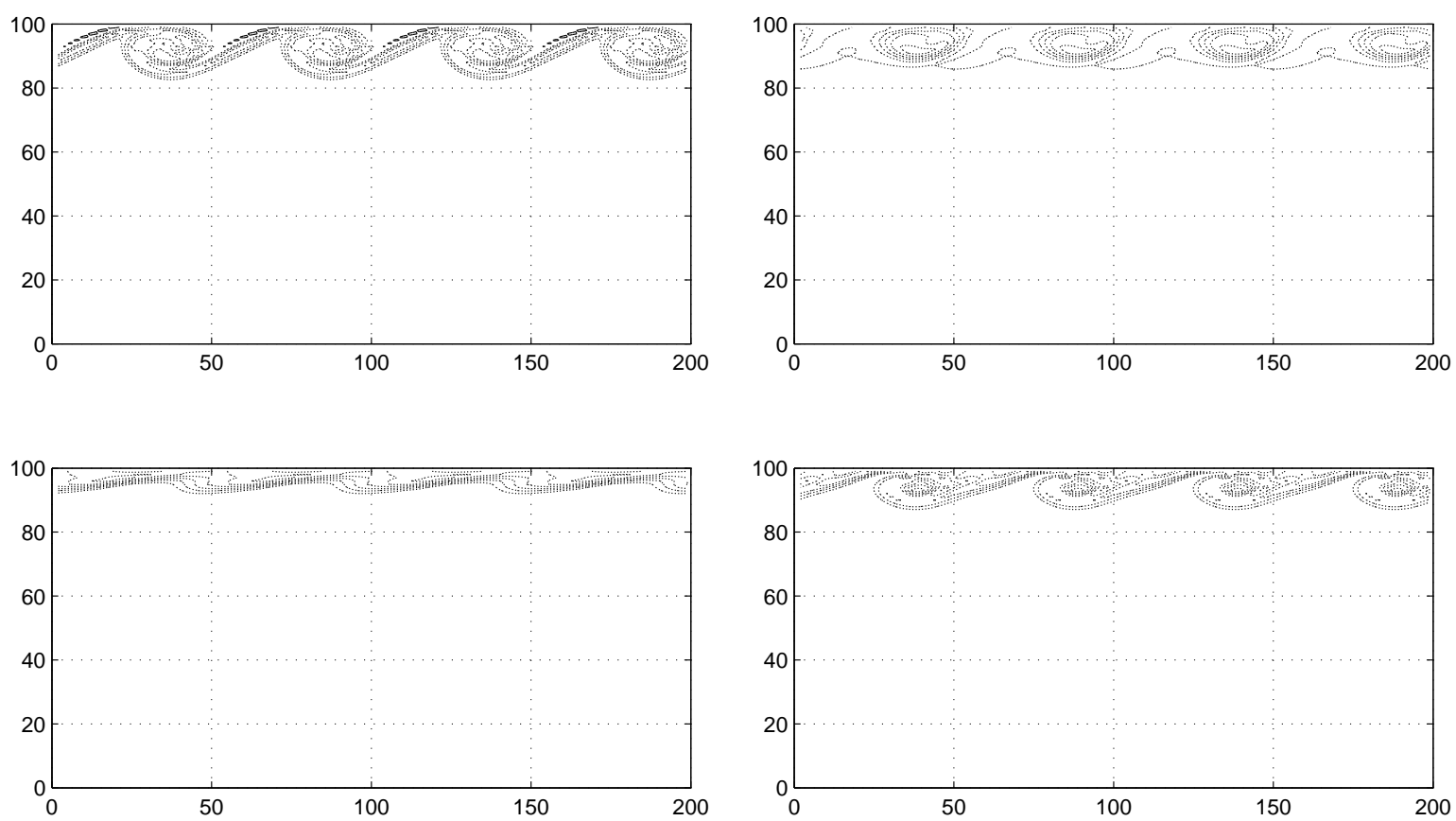

(a) $t=50$

(b) $t=100$
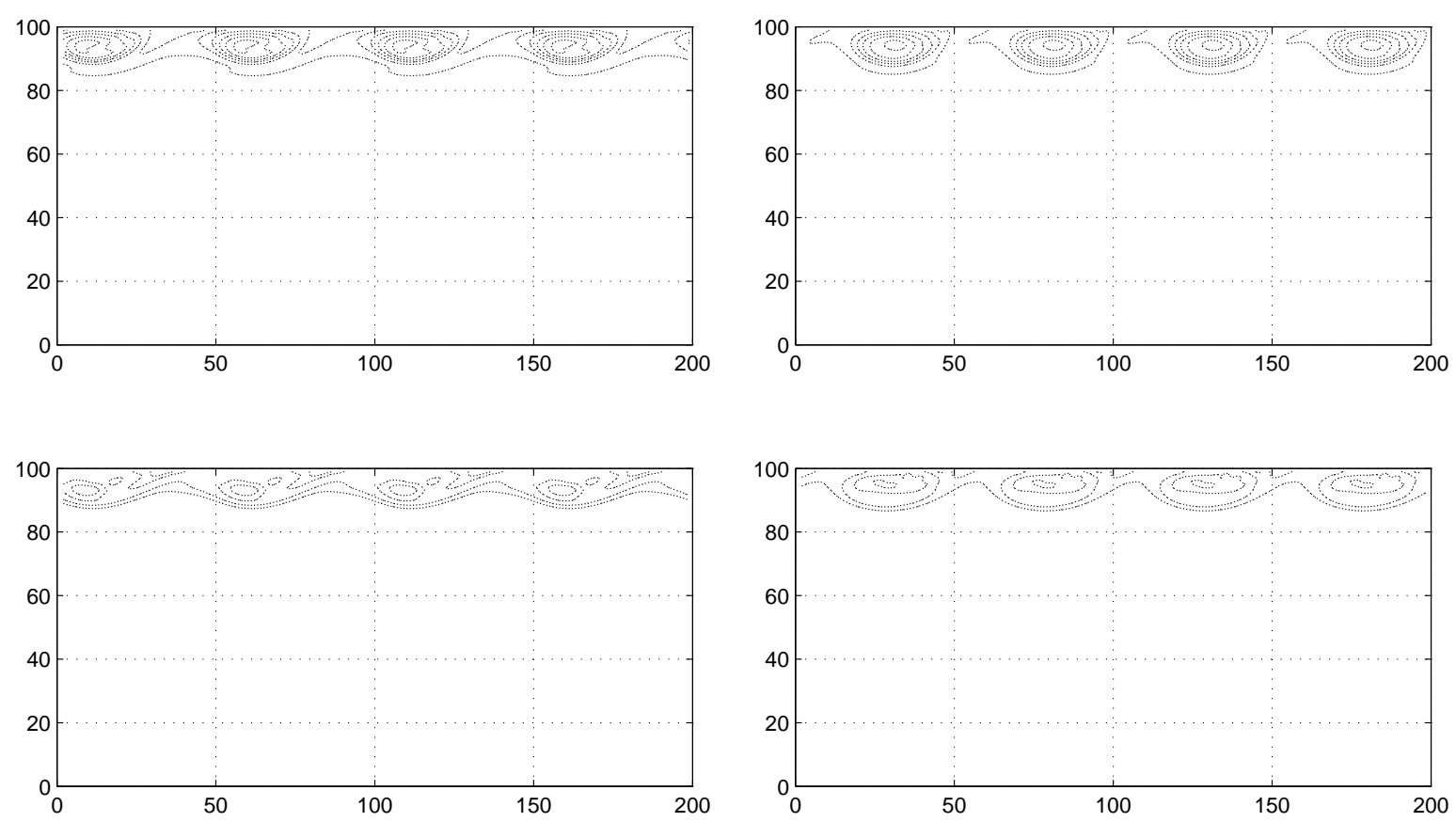

(c) $t=150$

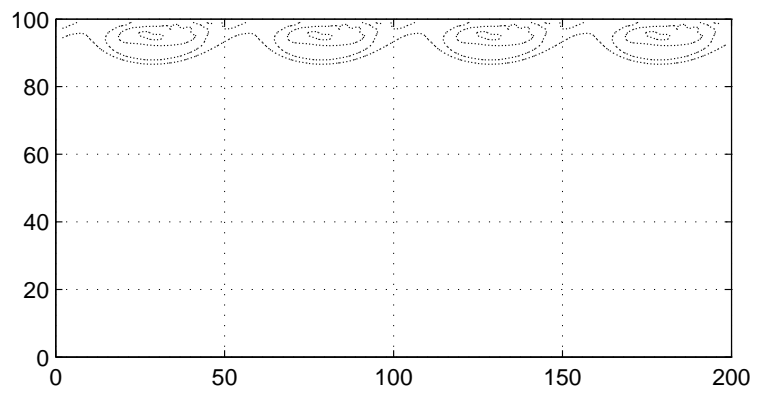

(d) $t=200$

Figure 10. Non-linear evolution of the potential vorticity in the upper layer (above) and in the lower layer (below) for regime B with $Q_{2} / Q_{1}=1$ and $L_{1} / R_{d}=1.0$. Positive/negative values of PV are represented by solid/dashed lines. 

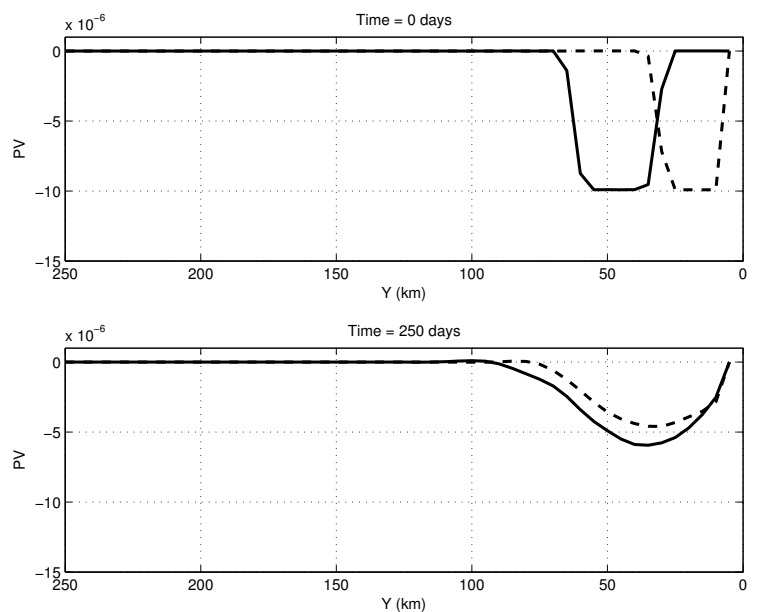

Figure 11. Zonal average of layerwise potential vorticity at the beginning and at 250 days in the simulation with $Q_{2} / Q_{1}=1$ and $L_{1} / R_{d}=1.0$; the solid (dashed) line corresponds to the upper (lower) layer. The widening of the PV profile corresponds to the effect of vortices. The deepening or the shallowing of the maxima is due to the zonal averaging process. 

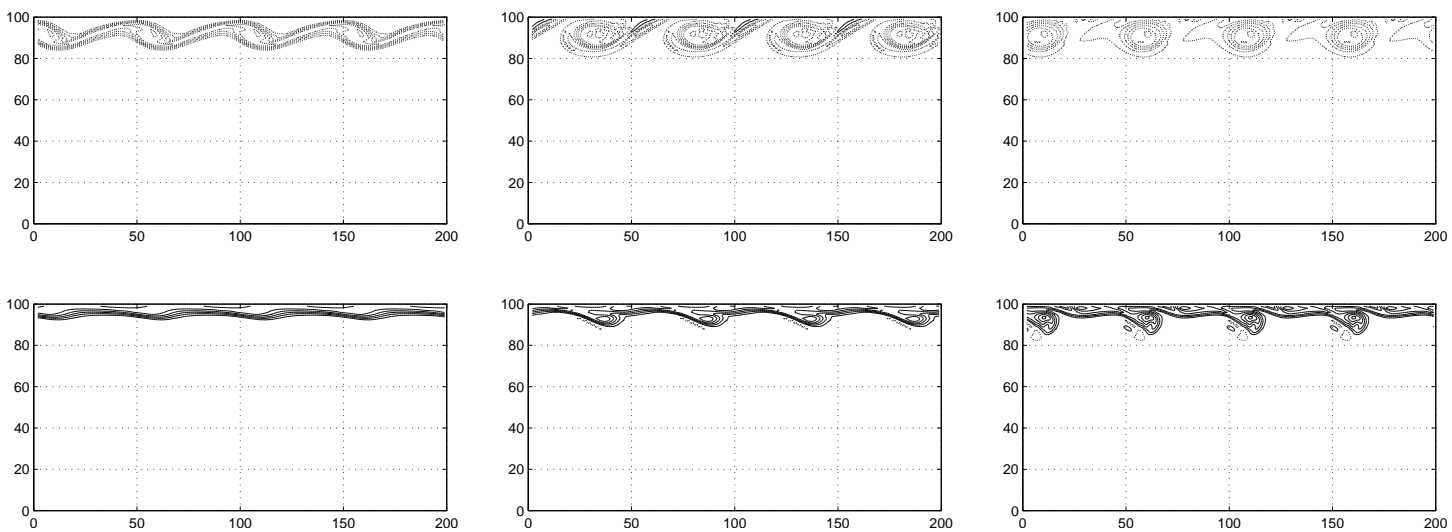

(a) $t=50$

(b) $t=75$

(c) $t=100$
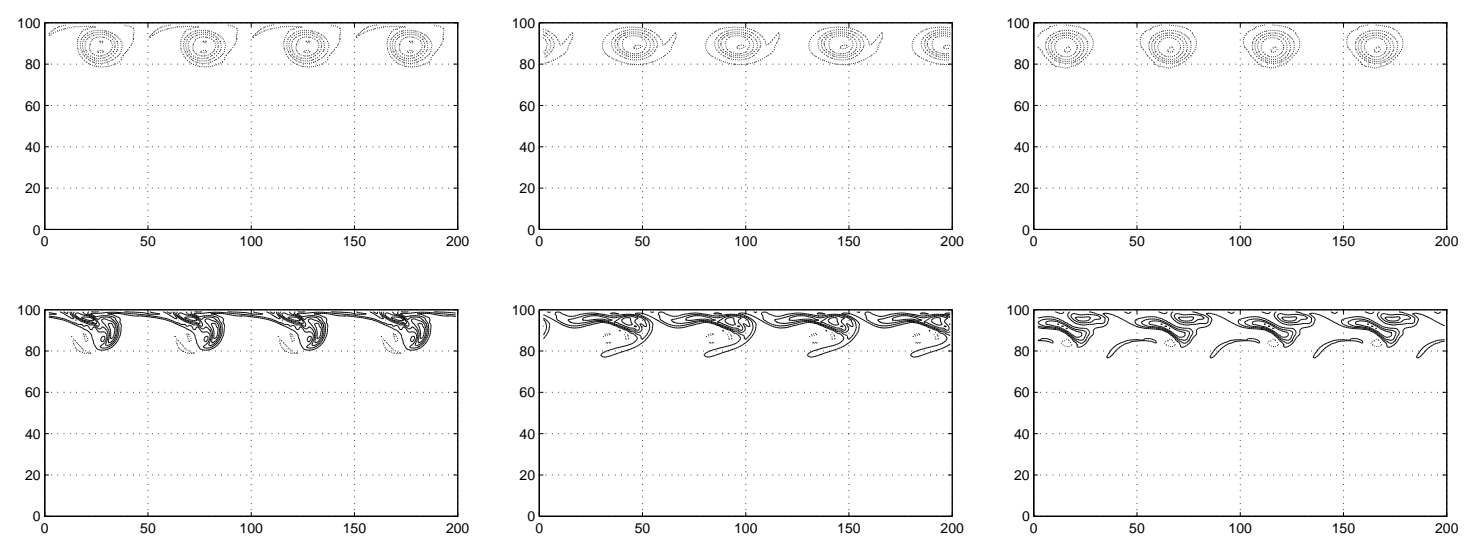

(d) $t=125$

(e) $t=150$

(f) $t=175$
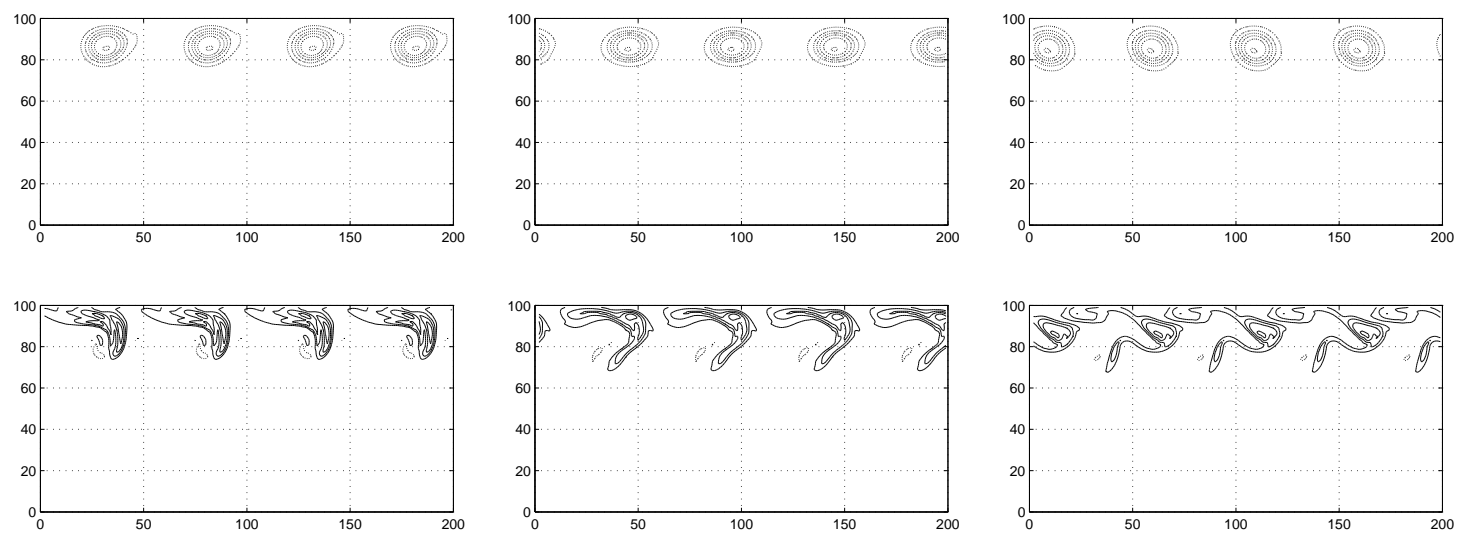

(g)t $=200$

(h) $t=225$

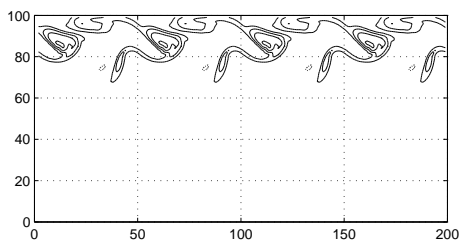

(i) $t=250$

Figure 12. Non-linear evolution of the potential vorticity in the upper layer (above) and in the lower layer (below) for regime $\mathrm{C}$ with $Q_{2} / Q_{1}=-1$ and $L_{1} / R_{d}=1.0$. Positive/negative values of $\mathrm{PV}$ are represented by solid/dashed lines. 


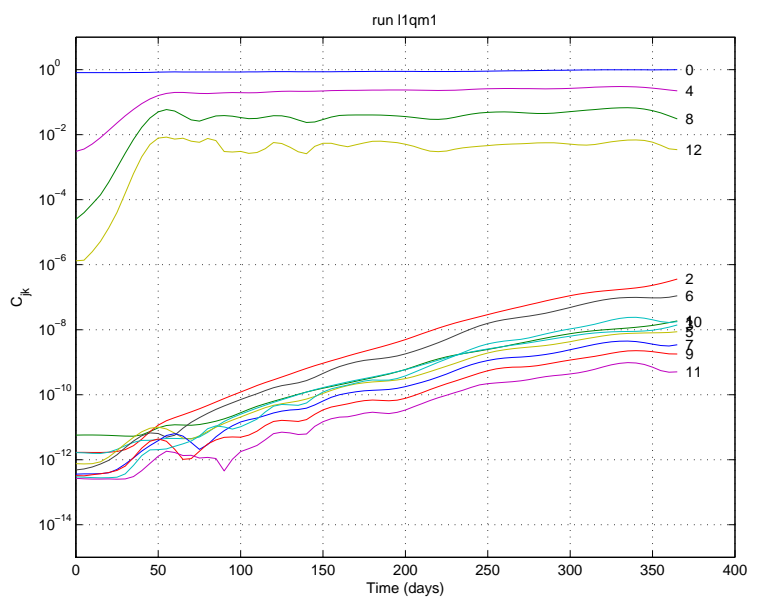

Figure 13. Fourier analysis along the zonal axis, of potential vorticity in the upper layer for regime $\mathrm{C}$ with $Q_{2} / Q_{1}=-1$ and $L_{1} / R_{d}=1$; the numbers next to each line are the wavenumbers. 

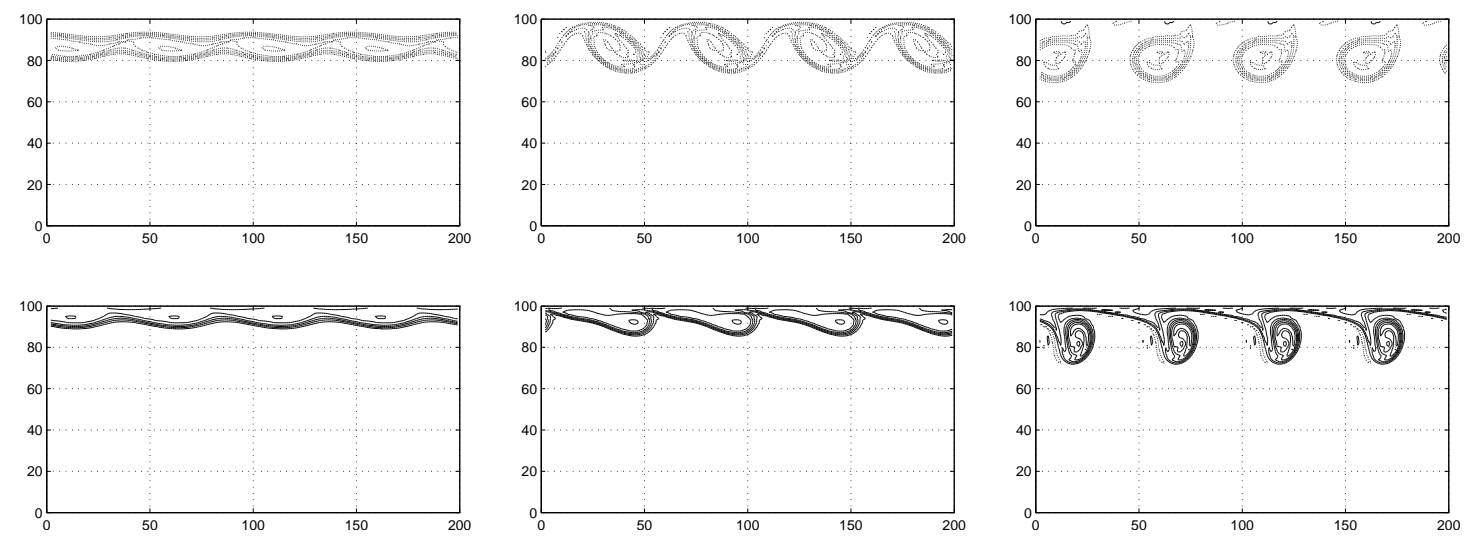

(a) $t=50$

(b) $t=75$

(c) $t=100$
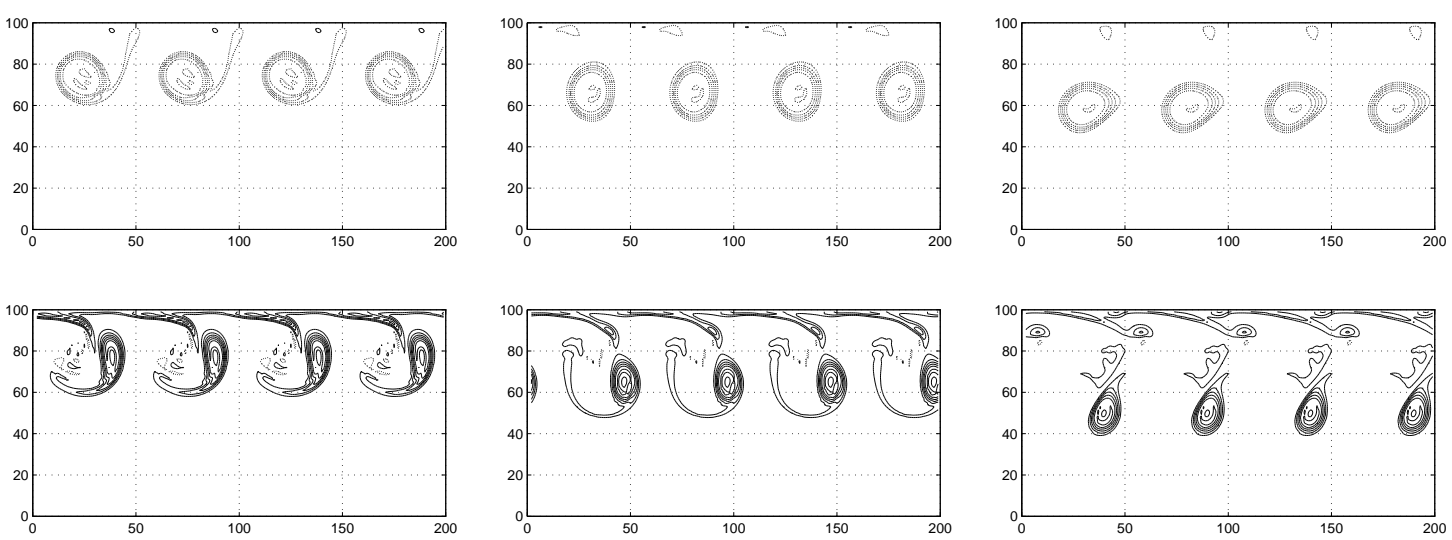

(d) $t=125$

(e) $t=150$

(f) $t=175$
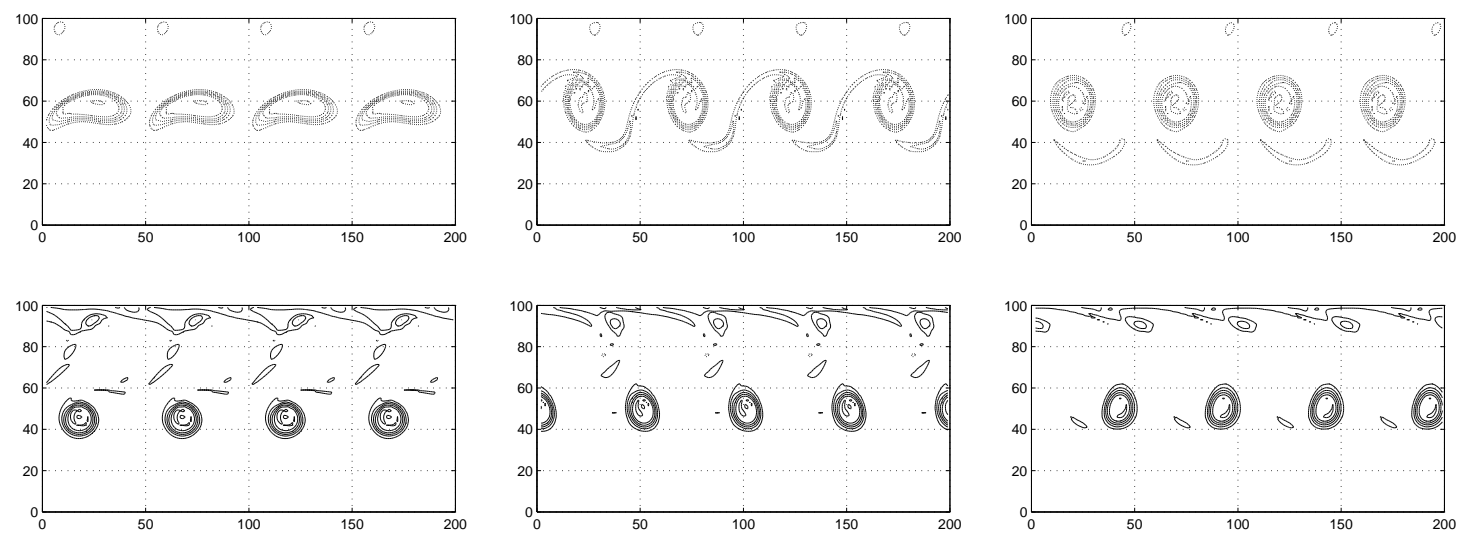

(g)t $=200$

(h) $t=225$

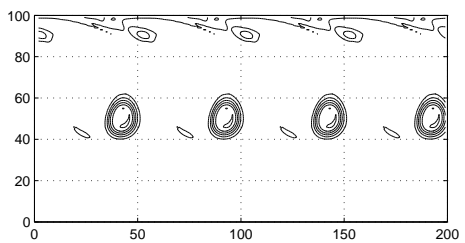

(i) $t=250$

Figure 14. Non-linear evolution of the potential vorticity in the upper layer (above) and in the lower layer (below) for regime $\mathrm{D}$ with $Q_{2} / Q_{1}=-1$ and $L_{1} / R_{d}=1.5$. Positive/negative values of $\mathrm{PV}$ are represented by solid/dashed lines. 

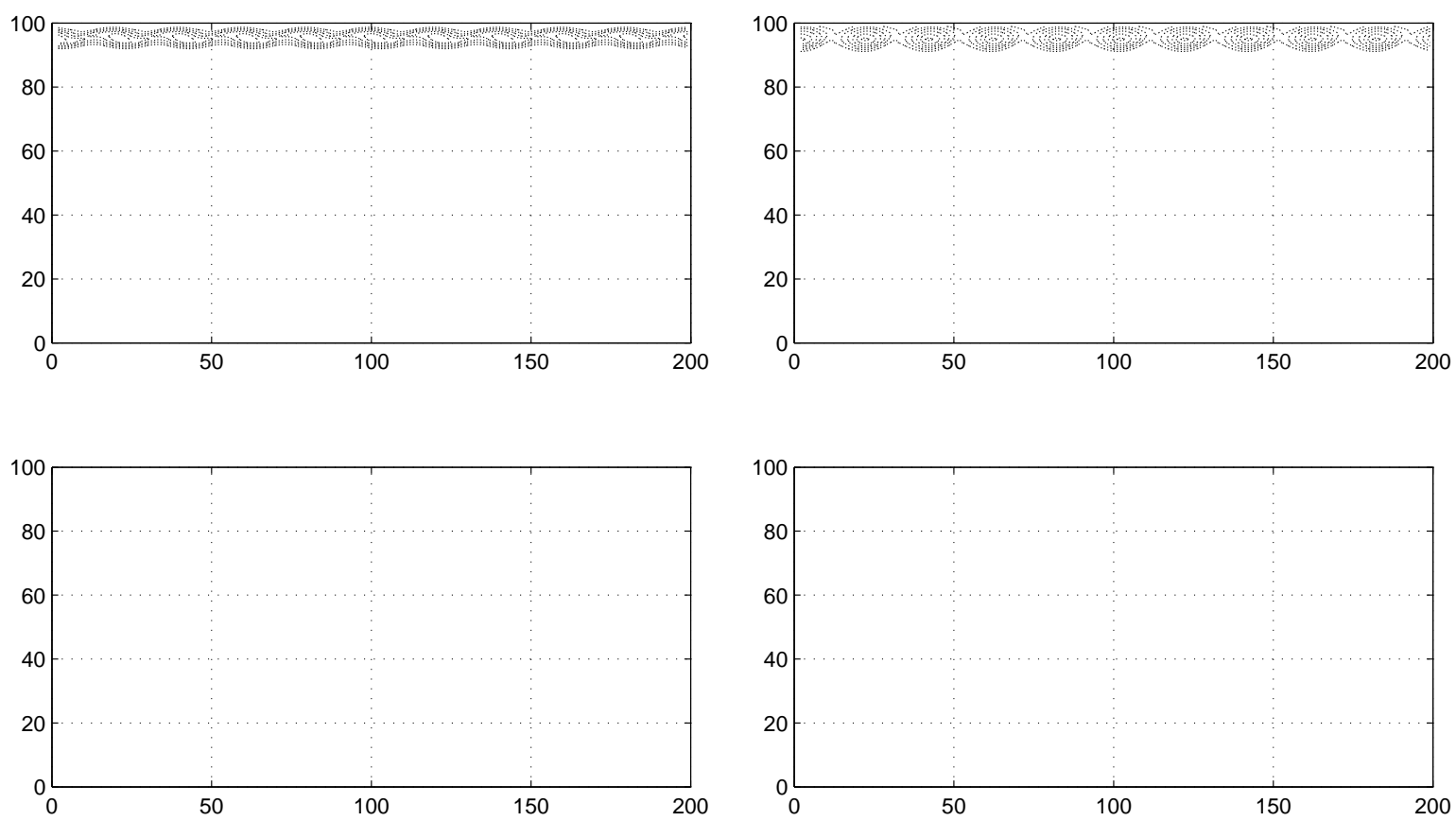

(a) $t=25$

(b) $t=50$
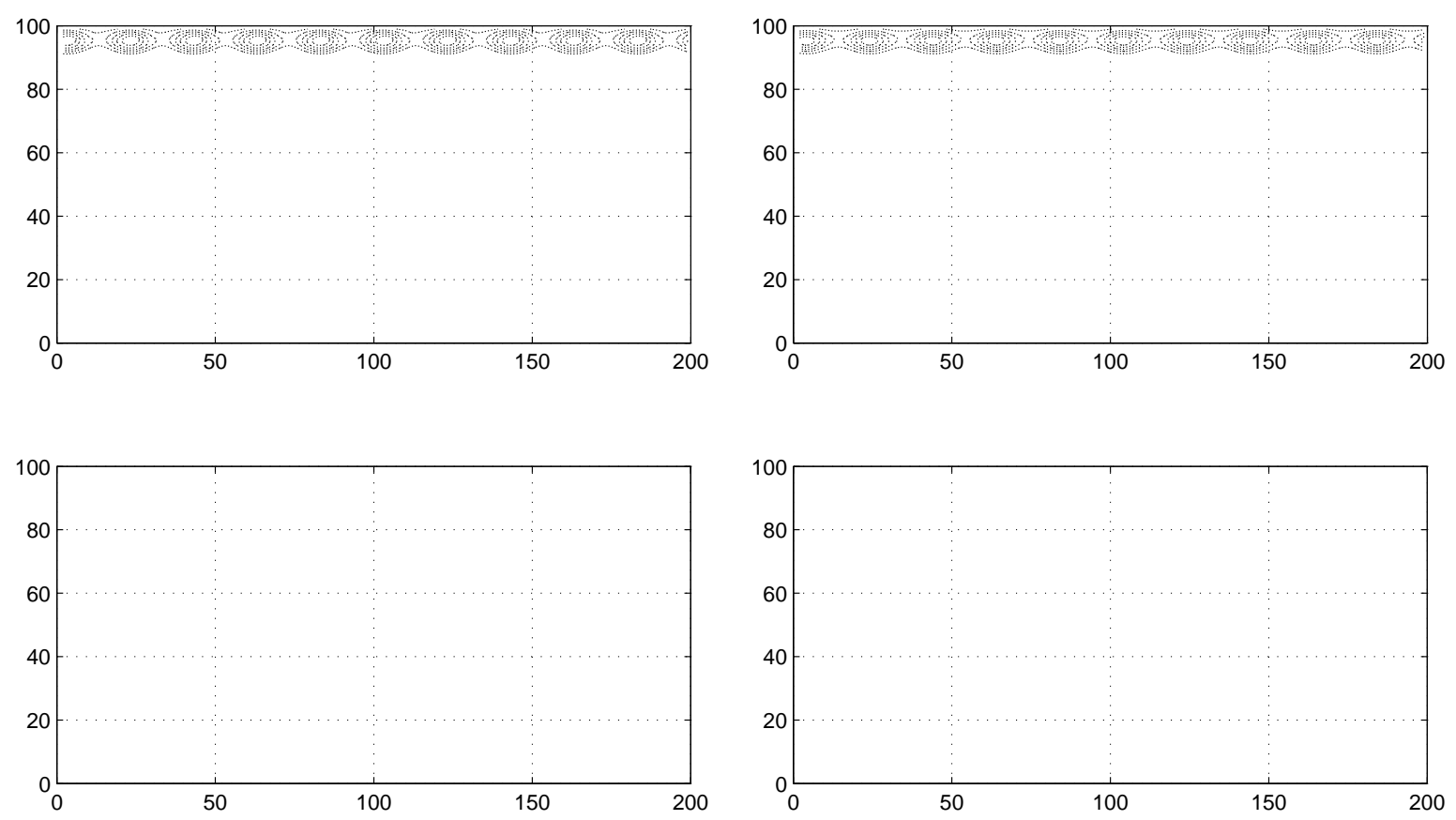

(c) $t=75$

(d) $t=100$

Figure 15. Non-linear evolution of the potential vorticity in the upper layer (above) and in the lower layer (below) for regime $\mathrm{E}$ with $Q_{2} / Q_{1}=0$ and $L_{1} / R_{d}=0.6$. Positive/negative values of $\mathrm{PV}$ are represented by solid/dashed lines. 

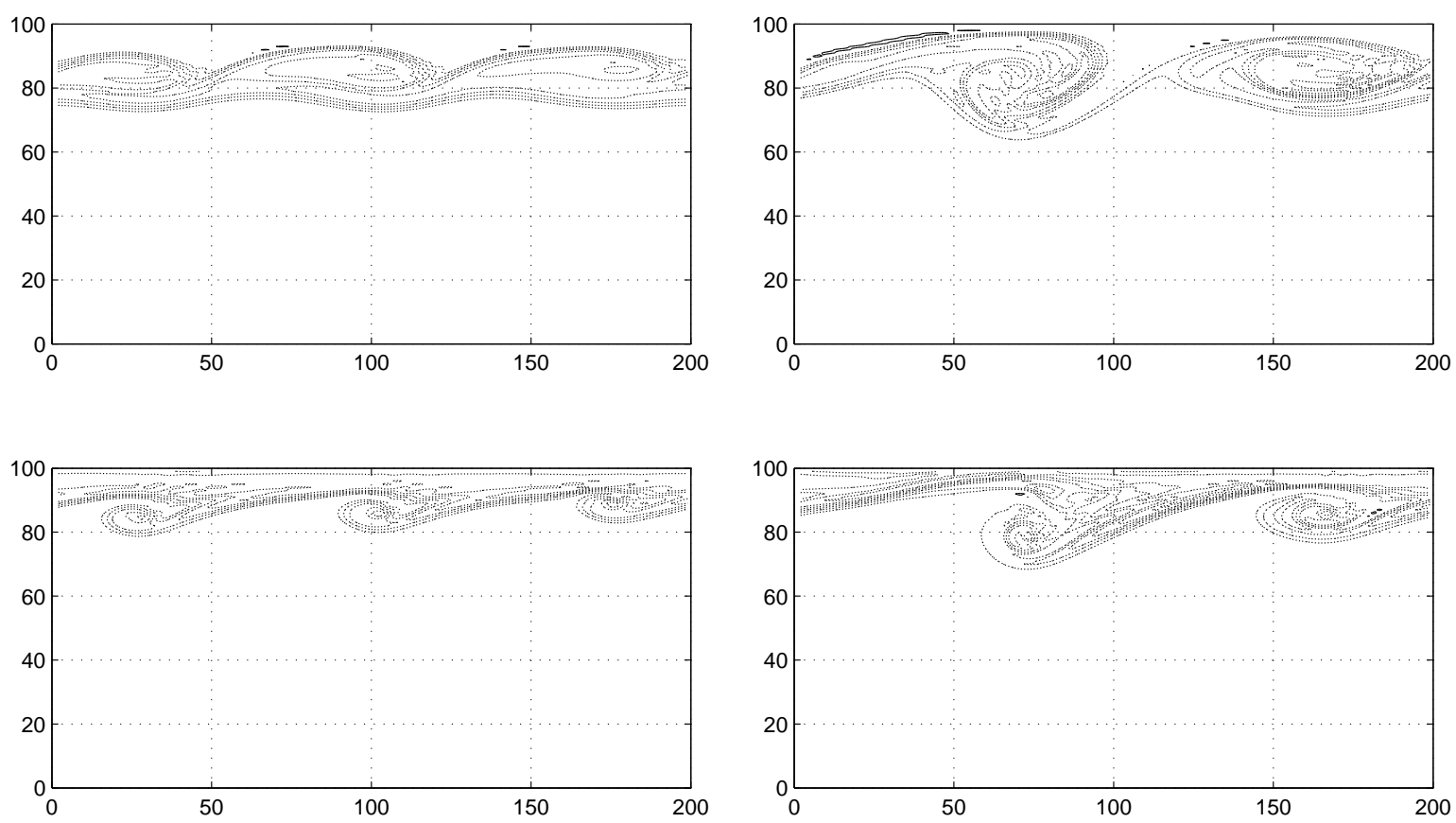

(a) $t=150$

(b) $t=175$
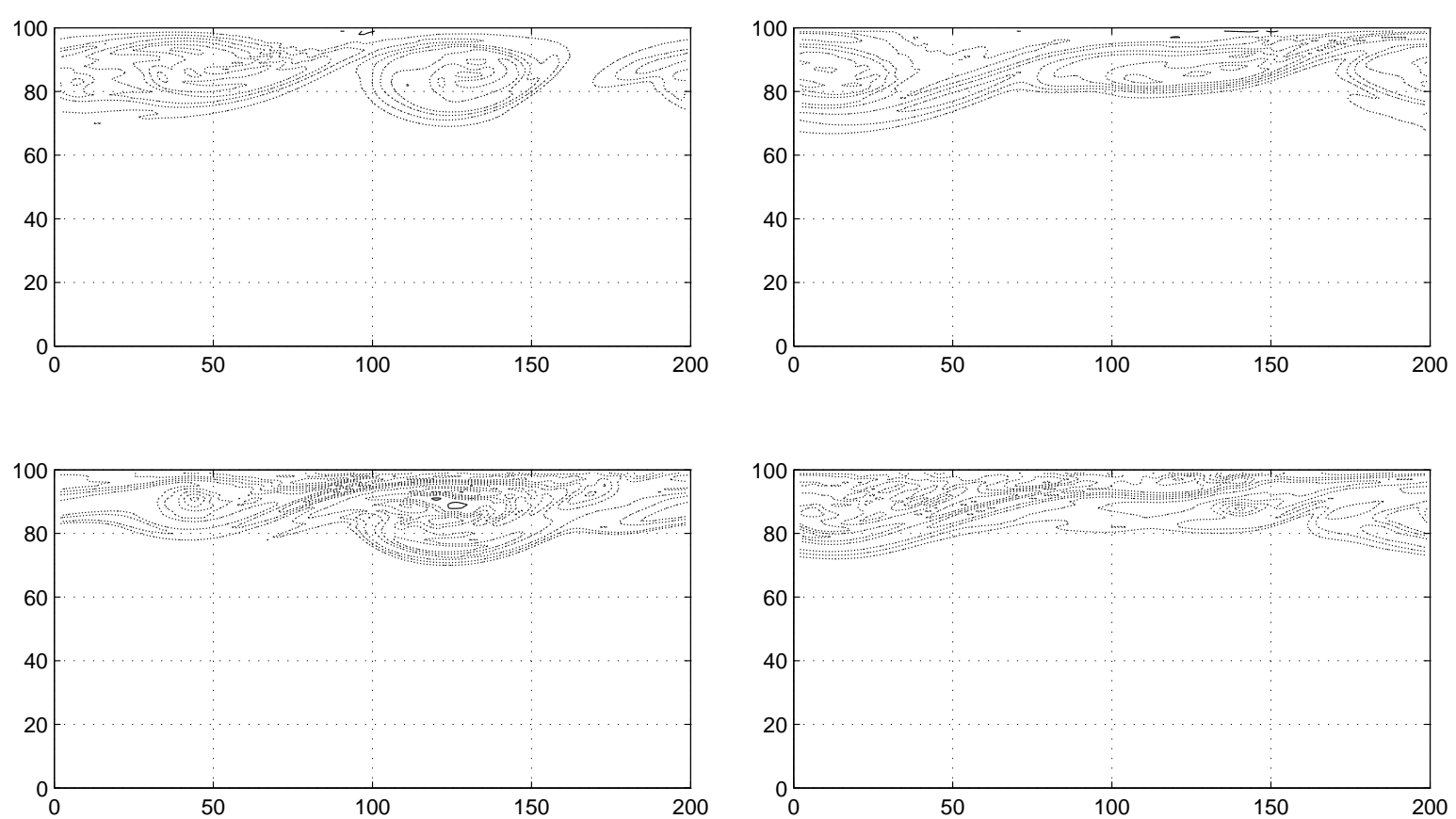

(c) $t=225$

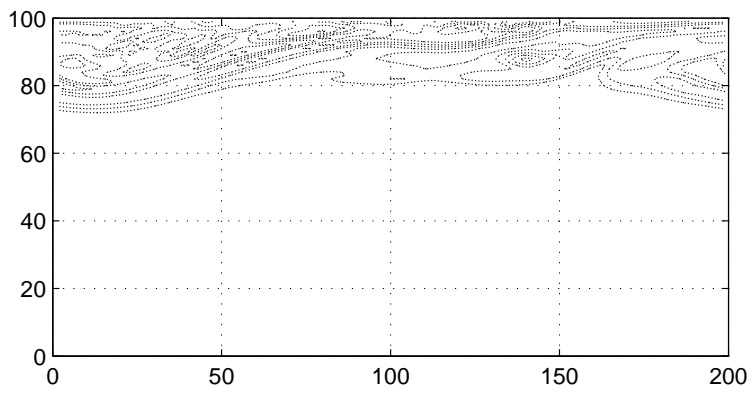

(d) $t=250$

Figure 16. Non-linear evolution of the potential vorticity in the upper layer (above) and in the lower layer (below) for regime $\mathrm{F}$ with $Q_{2} / Q_{1}=1$ and $L_{1} / R_{d}=2$. Positive/negative values of $\mathrm{PV}$ are represented by solid/dashed lines. 


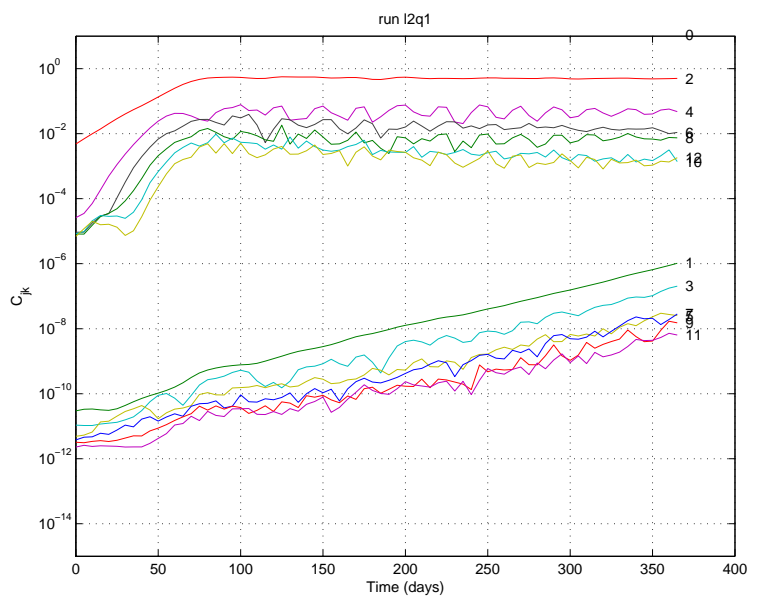

Figure 17. Fourier analysis along the zonal axis, of potential vorticity in the upper layer for regime $\mathrm{F}$ with $Q_{2} / Q_{1}=1$ and $L_{1} / R_{d}=2$; the numbers next to each line are the wavenumbers.

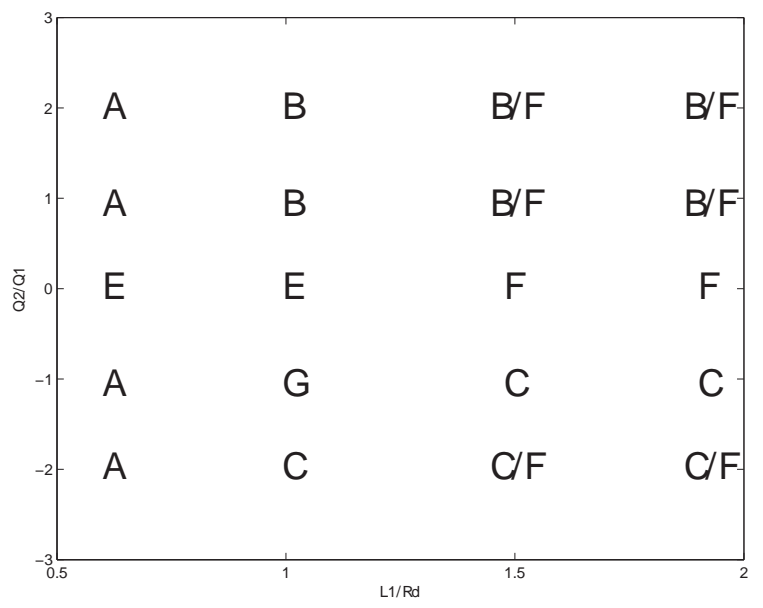

Figure 18. Classification of nonlinear regimes of flow with beta effect, in the $L_{1} / R_{d}, Q_{2} / Q_{1}$ plane. 


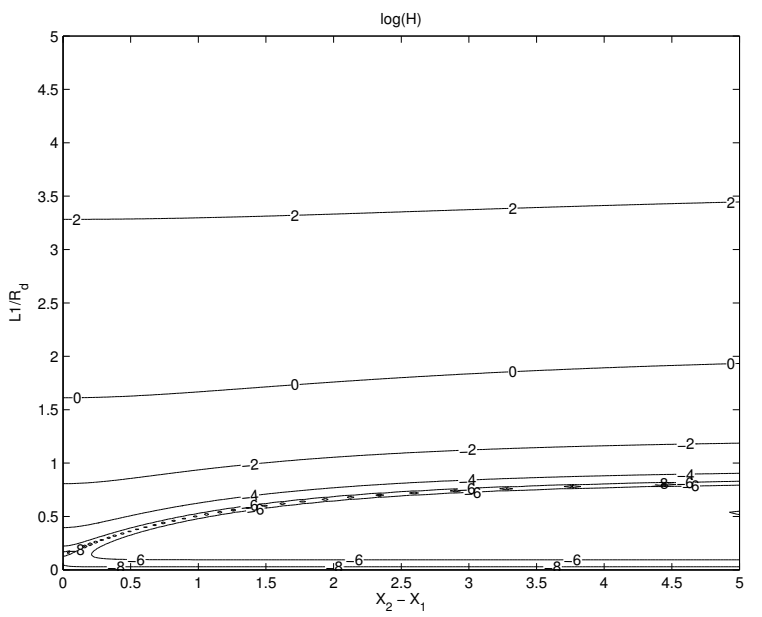

(a)

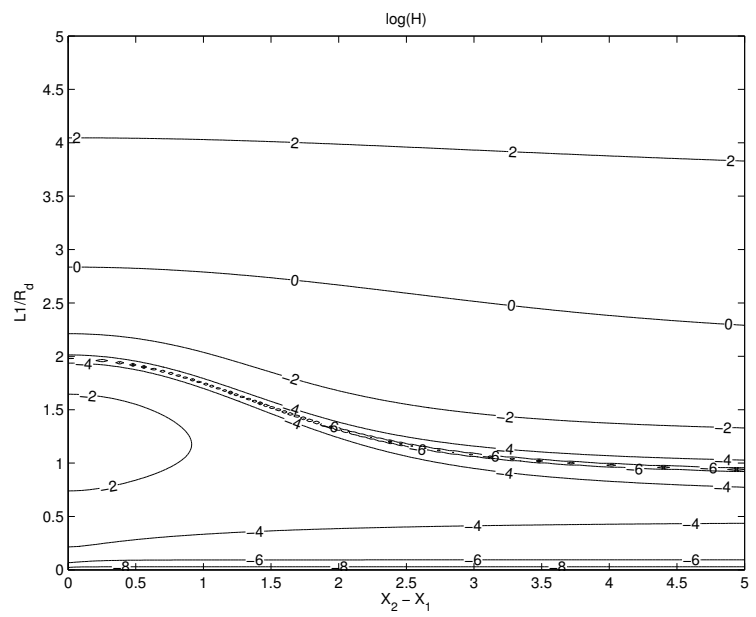

(b)

Figure 19. (a) Isolines of the Hamiltonian $H$ in the $\left(x_{2}-x_{1}, L_{1} / R_{d}\right)$ plane for $Q_{2} / Q_{1}=2$; (b) same as (a), now for $Q_{2} / Q_{1}=-2$. The dashed line is the local maximum of $H$, which separates the isolines of $H$ which close on themselves, at small $L_{1} / R_{d}$, from those which do not, at large $L_{1} / R_{d}$ 